\title{
L'industrie lithique du Paléolithique moyen récent de Roc de Combe (Payrignac, Lot, France), un nouvel exemple de Moustérien Discoïde à denticulés
}

The middle Palaeolithic lithic industry of Roc de Combe (Payrignac, Lot, France), A new example of Denticulate Discoïd Mousterian

Maria Lorenzo Martinez, Jean-Guillaume Bordes et Jacques Jaubert

\section{OpenEdition Journals}

Édition électronique

URL : http://journals.openedition.org/paleo/2769

DOI : $10.4000 /$ paleo.2769

ISSN : 2101-0420

Éditeur

SAMRA

Édition imprimée

Date de publication : 28 décembre 2014

Pagination : 101-124

ISSN : 1145-3370

Référence électronique

Maria Lorenzo Martinez, Jean-Guillaume Bordes et Jacques Jaubert, «L'industrie lithique du Paléolithique moyen récent de Roc de Combe (Payrignac, Lot, France), un nouvel exemple de Moustérien Discoïde à denticulés », PALEO [En ligne], 25 | 2014, mis en ligne le 20 août 2015, consulté le 07 juillet 2020. URL : http://journals.openedition.org/paleo/2769; DOI : https://doi.org/10.4000/ paleo. 2769

\section{(c) $)(9)$}

PALEO est mis à disposition selon les termes de la licence Creative Commons Attribution - Pas d'Utilisation Commerciale - Pas de Modification 4.0 International. 


\title{
L’industrie lithique du Paléolithique moyen récent de Roc de Combe (Payrignac, Lot, France), un nouvel exemple de Moustérien Discoïde à denticulés
}

\author{
Maria LORENZO MARTINEZ(1), Jean-Guillaume BORDES ${ }^{(2)}$, \\ Jacques JAUBERT ${ }^{(2)}$
}

\begin{abstract}
Résumé : Roc de Combe est l'un des sites de référence pour étudier les modalités de passage Paléolithique moyen récent - Paléolithique supérieur initial (Châtelperronien) et Paléolithique supérieur ancien (Aurignacien ancien) dans le Sud-Ouest de la France. Fouillé par F. Bordes en 1966 et longtemps réputé pour une « interstratification »- désormais rejetée - servant la thèse de l'acculturation, il a fait l'objet d'une étude des niveaux attribués au Châtelperronien et à l'Aurignacien, mais les séries du Moustérien demeuraient jusqu'à ce jour identifiées, cependant non étudiées. Un récent travail universitaire mené par l'une d'entre nous (M.L.M.) permet désormais de disposer d'un bilan techno-économique et typologique confirmant l'attribution de cette série au Moustérien de débitage Discoïde à denticulés, techno-complexe qui clôt dans le Sud-Ouest de la France nombre d'archéo-séquences. La présente étude donne les premières données numériques relatives à cette série inédite, la positionne dans un cadre chrono-culturel et son contexte régional, en insistant sur un statut pétrographique relativement original propre à la Bouriane, région faisant la liaison entre Périgord et Quercy.
\end{abstract}

Mots-clés : Paléolithique moyen récent, stade isotopique 3, Discoïde, Moustérien Discoïde à denticulés, Périgord, Quercy, matières premières, techno-économie lithique.

Abstract: The middle Palaeolithic lithic industry of Roc de Combe (Payrignac, Lot, France), A new example of Denticulate Discoïd Mousterian. Roc de Combe (Lot, France) is one of the key sites concerning the Late Middle to Upper Palaeolithic transition in southwestern France. 1966' excavation, led by François Bordes, has yielded some Mousterian, Chatelperronian, Aurignacian and Gravettian rich assemblages. Here we present the analysis of the unpublished Mousterian lithic assemblages, studied by one of us during a Master degree. From this study, the belonging of this industry to a Denticulate-Discoid Mousterian has been confirmed, a technocomplex usually classically attributed to the final stage of the Mousterian. Several radiocarbon dates confirm this attribution. Finally, our analysis shows an original lithic raw material procurement strategy, due to the site location, peculiar to the Bouriane between Perigord and Quercy.

Key-words: Late Middle Paleolithic (LMP), Isotopic stage 3 (MIS 3), Discoid, Denticulate Mousterian, Perigord, Quercy, lithic technology, raw material.

(1) 39 rue de la Garde, FR-63000 Clermont-Ferrand - lamaruxina@gmail.com

(2) PACEA UMR 5199 CNRS, Université de Bordeaux, Bâtiment B8 Allée Geoffroy Saint-Hilaire CS 50023 FR-33615 Pessac Cedex jg.bordes@pacea.u-bordeaux1.fr ; j.jaubert@pacea.u-bordeaux1.fr 


\section{1 - Introduction}

La caractérisation techno-économique et le positionnement chronologique des derniers techno-complexes moustériens du Sud-Ouest de la France est un thème de recherche prisé et dynamique depuis une dizaine d'années (e.g. Soressi 2002 ; Slimak 2004 ; Lahaye 2005 ; Thiébaut 2005 ; Jaubert et al. 2011 ; Gravina et al. 2012 ; Richter et al. 2012). Notamment parce qu'il porte en filigrane l'enjeu de statuer sur leur relation - et celles de leurs auteurs, les ultimes Néandertaliens - avec les premières cultures du Paléolithique récent, Châtelperronien et Aurignacien. L'étude du gisement du Roc de Combe (Payrignac, Lot) ${ }^{3}$ entre parfaitement dans cette dynamique puisque, suite à l'étude des niveaux du Paléolithique supérieur initial (Châtelperronien) et ancien (Bordes 2002), l'intérêt s'est porté récemment sur les niveaux moustériens inédits (Lorenzo Martinez 2012).

L'analyse de ce matériel a été effectuée dans un contexte favorable pour différentes raisons. Tout d'abord, les collections de référence sont conservées au Musée National de Préhistoire (Les Eyzies-de-Tayac). Comme d'autres, elles ont vocation à faire l'objet de réexamens, lesquels justifient l'utilisation de méthodes modernes de laboratoire (taphonomie, archéozoologie, archéopétrographie, technologie lithique ou de la matière dure animale, remontages...), voire de terrain (géoarchéologie, datations...). Dans ce contexte, les séries du Roc de Combe n'avaient été en effet que partiellement évoquées, le plus souvent par François Bordes et son équipe, désireux de contribuer à différentes synthèses régionales (Bordes 1972, 1981 ; Grayson et Delpech 2008). Dans le même temps, plusieurs archéoséquences moustériennes majeures du Périgord et de Charente ont été réinvesties à ce titre, voire à nouveau documentées récemment (La Quina, Fontéchevade, Combe-Capelle, Pech de l'Azé I, IV, Roc-deMarsal, La Ferrassie...). Le Moustérien de Roc de Combe s'inscrit dans cette revalorisation puisque son étude résulte d'une volonté conjointe de publier cette importante séquence afin de pouvoir l'intégrer dans les synthèses régionales du Moustérien du bassin Aquitain (e.g. Turq et al. 2008) et, plus globalement, de l'Europe du Sud-Ouest.

Quelques diagnostics sur la série issue des niveaux moustériens ont été effectués avant la présente contribution. En premier par François Bordes lui-même, qui publia une courte note dans ses « Leçons sur le Paléolithique " (Bordes 1984) où il suggère une attribution incertaine au Moustérien de tradition acheuléenne (MTA). L'un de nous écrivait à propos de la zone au-dessus de l'effondrement ou séquence classique (I à $K$ ) de Roc de Combe : « [à l'intérieur de la grotte] le Moustérien (« 9 » ou
« $M$ » ou encore «Moustérien du haut » des sacs et carnets de fouilles) est pauvre dans cette zone, la fouille s'étant arrêtée à son sommet à cause des gros blocs d'effondrement. II se distingue nettement des deux industries précédentes par l'importance du débitage sur quartzite, matière absente de couches sus-jacentes (...) ; par la typologie (racloirs et denticulés) ; par le débitage (discoïde très majoritaire, mais aussi quelques traces de Levallois). » (Bordes J.-G. 2002, p. 68). Et, plus loin, à propos de la zone 2 (pente : base de $\mathrm{A}$ à $\mathrm{E}$ ) : "Les observations suivantes devront être quantifiées par une analyse plus systématique. On peut néanmoins déjà remarquer que ce matériel correspond à une industrie moustérienne homogène, identique à celle décrite pour la partie haute du gisement (...) : débitage discoïde (Boëda 1993) très nettement dominant, orienté vers la production de pointes pseudo-Levallois, rarement retouchées en racloirs transversaux ou doubles déjetés. De nombreux denticulés ont été réalisés sur éclats épais par coches clactoniennes adjacentes. Quelques éclats Levallois sont présents, souvent en silex sénonien de bonne qualité. Le reste du débitage est essentiellement réalisé à partir de silex tertiaire, de qualité moyenne et nettement inférieur à ceux exploités au Châtelperronien et à l'Aurignacien du gisement. Une autre différence avec les industries du Paléolithique supérieur est la part importante du débitage sur quartzite. Typologiquement, cette industrie est très certainement un Moustérien à denticulés, à débitage non Levallois. 》 (op. cit. p. 70)

Ce diagnostic se devait donc d'être prolongé par une étude plus précise et chiffrée en vue d'une caractérisation étayée, et ainsi positionner la série dans le cadre du Moustérien de débitage Discoïde 4 à denticulés récemment revu (Thiébaut 2005, 2007) ; puis, entre autres, contribuer via ce Moustérien de Roc de Combe à étayer le tableau des archéo-séquences du Sud-Ouest de la France, dans lesquelles ce techno-complexe lithique (TCL) prend place après le MTA, au dessus des TCL Levallois, Quina (Turq et al. 2008 ; Turq, Jaubert 2008) et après le MTA (Jaubert 2010, 2012).

\section{2 - Présentation du site et de la série étudiée}

\section{1 - Contextes géographique et géologique}

Roc de Combe (Payrignac, Lot) est localisé sur la bordure orientale du Bassin aquitain, aux confins du Périgord (Dordogne) et de ses formations du Crétacé supérieur d'une part, du Quercy (Lot) et ses âpres plateaux

(3) F. Bordes utilise indifféremment la mention « le gisement de Roc de Combe » (titre) ou « du Roc de Combe » (texte). Henri Laville a opté pour la seconde option tandis que le Dictionnaire de la Préhistoire dirigé par A. Leroi-Gourhan (PUF 1988) respecte le titre de l'article princeps de 1967.

(4) À l'initiative de V. Mourre, on met désormais une majuscule à "Discoïde " quand on qualifie une méthode de débitage définie par Boëda (1993) : "Le terme "Discoïde » ne sera jamais employé ici au sens qualificatif de "en forme de disque " mais renvoie toujours à un schéma de production ; il est donc légitime de parler de conception Discoïde du débitage, la majuscule mettant l'accent sur la nuance. " (Jaubert et Mourre 1996 - note 1, p. 338). 
jurassiques d'autre part, en rive sud de la Dordogne, à une dizaine de kilomètres au nord-ouest de Gourdon (1 ${ }^{\circ} 20^{\prime} 45 \mathrm{E} ; 44^{\circ} 46^{\prime} 18 \mathrm{~N}, 150 \mathrm{~m}$ NGF) (fig.1). La petite grotte s'ouvre au pied d'une butte de calcaire coniacien, surmontée d'altérites tertiaires, formation localement dénommée pech. II s'agit d'une modeste cavité prolongée latéralement par un abri, s'ouvrant au sud sur un vallon sec, lui-même affluent de la Dordogne dont la vallée se situe à $6 \mathrm{~km}$ au nord. C'est donc une zone géographique, géologique et écologique de transition (écotone) entre les mondes périgourdin à l'ouest et quercinois à l'est.

\section{2 - Historique des travaux}

En 1950, Jean Labrot découvre la grotte et achète la parcelle, puis effectue un sondage en 1959. C'est l'intérêt de la séquence révélée par ces premiers travaux, couvrant une large tranche de temps - du Moustérien au Gravettien - qui incita F. Bordes et son équipe à y effectuer des fouilles, durant les trois mois de l'été 1966, conjointement avec l'inventeur et propriétaire. À l'issue de la première campagne, un différend entre les deux hommes mit rapidement un terme à la fouille. Le gisement fut d'abord publié sous forme d'une note (Bordes et Labrot 1967), puis intégré à une synthèse sur le «Périgordien »
(Bordes 1968). Dans le même temps que les travaux menés par $\mathrm{F}$. Champagne et $\mathrm{R}$. Espitalié sur le site voisin du Piage (Fajoles, Lot : Champagne et Espitalié 1967), F. Bordes y repéra sa fameuse "interstratification" entre Châtelperronien (à l'époque «Périgordien inférieur ») et Aurignacien, étayant le modèle alors controversé de lignées parallèles entre "Périgordien » et Aurignacien (Peyrony 1933 ; Bordes 1968 ; Rigaud 2000 ; Klaric 2003). Ces travaux permirent de mettre au jour une intéressante séquence s'échelonnant du Moustérien récent au Paléolithique supérieur: Châtelperronien, Aurignacien et Gravettien (fig. 2).

Henri Laville intégra la séquence de Roc de Combe dans le cadre de sa thèse à laquelle il consacra une longue notice même si la base de la séquence avec le(s) niveau(x) moustérien(s) n'est qu'entrevue (Laville 1969). Contrairement à nombre de séquences régionales, Roc de Combe ne fit alors pas l'objet d'une révision géologique avec des concepts et méthodes réactualisés comme celles de J.-P. Texier (Texier 2009).

La fouille fut menée selon une stratégie habituelle dans un tel contexte géomorphologique : établissement d'une tranchée perpendiculaire à la falaise, du talus jusqu'à

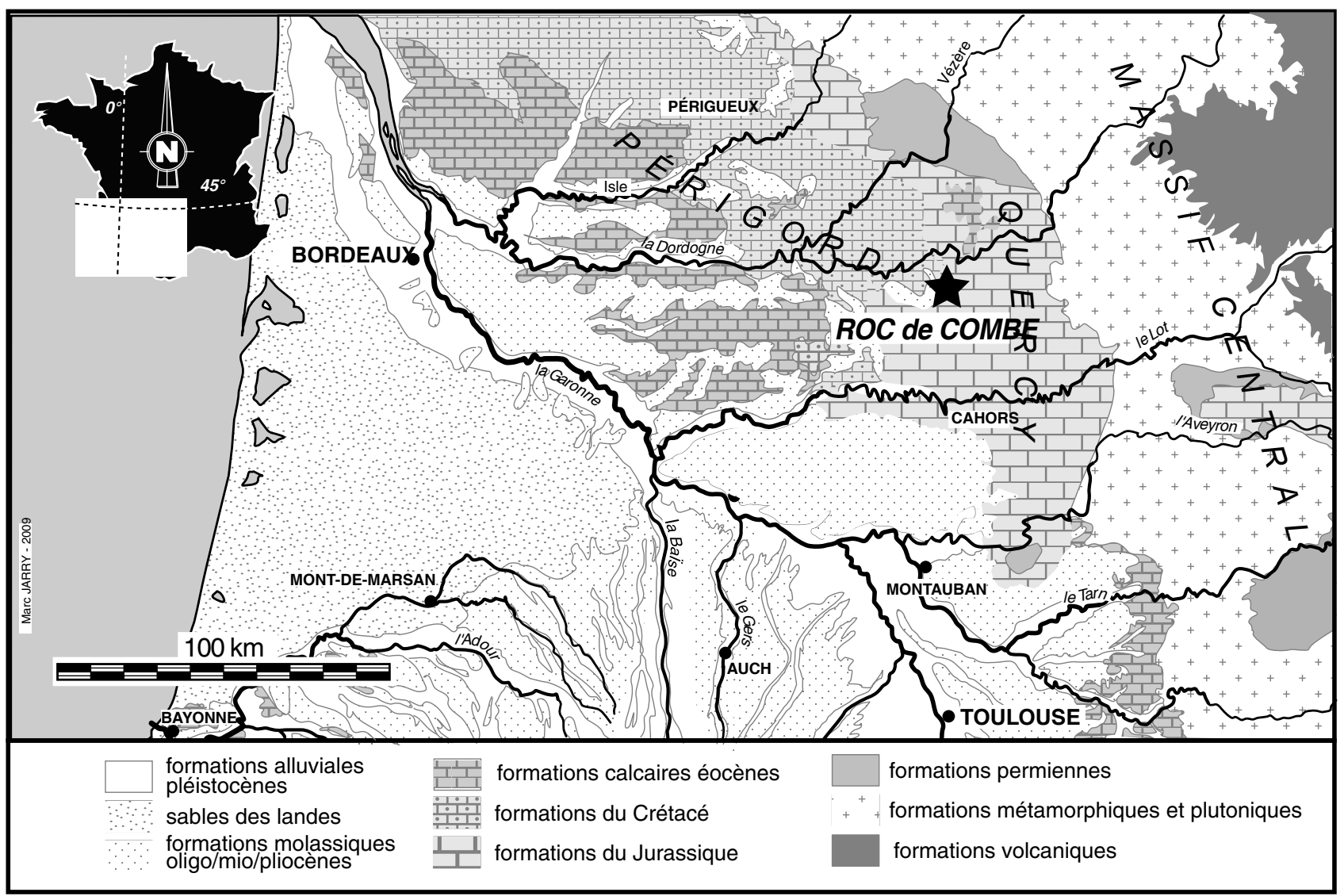

Figure 1- Localisation géographique de Roc de Combe (DAO M. Jarry, modifiée).

Figure 1- Location of Roc de Combe. 


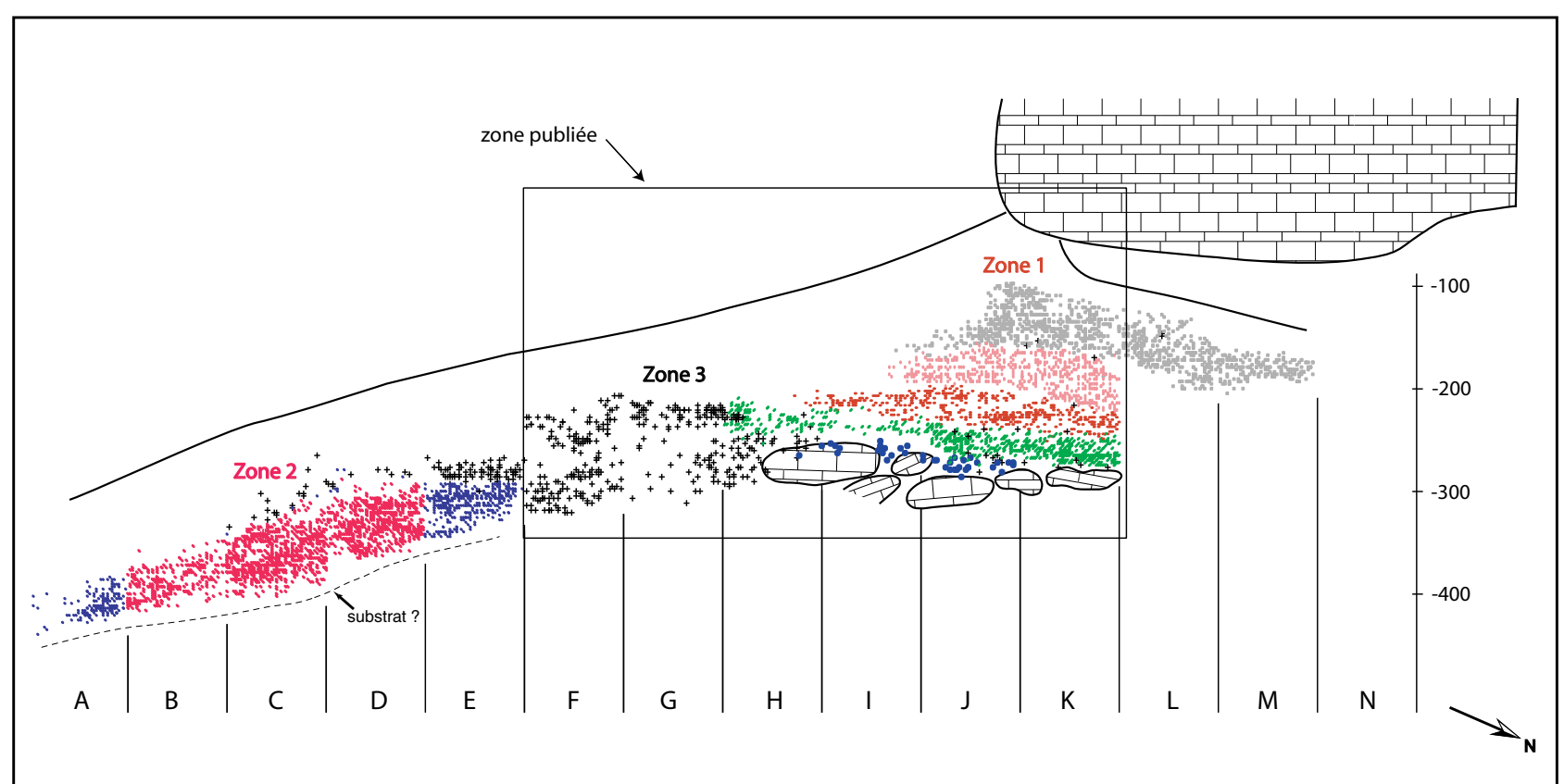

\begin{tabular}{|ll|}
\hline \multicolumn{1}{|c|}{ Légende : } \\
\hline$+\quad \begin{array}{l}\text { pièces dont l'attribution chrono-culturelle a varié } \\
\text { entre le moment de la fouille et leur marquage }\end{array}$ \\
\hline $\begin{array}{l}\text { pièces dont l'attribution chrono-culturelle faite à } \\
\text { la fouille et celle du marquage sont identiques : }\end{array}$ \\
- C.1 à C.4 : Gravettien \\
I $\quad$ C.5 à C.6: : Aurignacien récent \\
$-\quad$ C.7:Aurignacien ancien \\
$-\quad$ c.8:Châtelperronien \\
$-\quad$ C.B.:Moustérien \\
- Moustérien "du haut"
\end{tabular}

Figure 2 - Roc de Combe (Lot). Projection sagittale des pièces cotées avec délimitation des trois zones, Roc de Combe (Lot), (Bordes 2002, modifié).

Figure 2 - Roc de Combe (Lot). Sagittal projection of coordinated objects and areas 1,2 and 3 delimitation.

l'intérieur de la grotte, totalisant ainsi une vingtaine de mètres carrés (Bordes et Labrot 1967 ; Grayson et Delpech 2008) (fig. 3). Pour les niveaux moustériens notamment, une partie des pièces a été cotée en trois dimensions tandis que la majorité des vestiges n'ont pas été enregistrés ou simplement recueillis par couche et mètres carrés. Le seuil de cotation était la dimension, évaluée subjectivement par chaque fouilleur (com. pers. J.-Ph. Rigaud). Pour le Moustérien, et au contraire du Paléolithique supérieur, aucun tamisage n'a été effectué ; de ce fait, la fraction inférieure à $6 \mathrm{~mm}$ est absente des séries. Au moins pour certains carrés, notons cependant que la fouille a été menée de façon assez moderne, puisque plus de 4000 objets lithiques et éléments de faune ont été coordonnés au sein des niveaux moustériens.

Une première réévaluation de la séquence a été réalisée par J.-Ph. Rigaud (2000), puis par l'un de nous (Bordes 2002), cette dernière permettant de définir trois zones se distinguant par leur histoire sédimentaire et leur contenu archéologique (Zone 1 / séquence classique ; Zone 2 / Moustérien et, entre ces deux dernières la Zone 3 / Interstratification). L'analyse taphonomique des séries,
Gravettien exclu, a contribué à remettre en cause cette fameuse interstratification, la zone où elle fut définie n'ayant livré aucun niveau en position primaire cohérent. De plus, cette étude a montré que l'on pouvait distinguer, en première analyse, deux ensembles moustériens à Roc de Combe (fig. 2) :

- un modeste lot de pièces d'allure moustérienne provenant de la zone 1, directement sous-jacent au Châtelperronien, au niveau et en arrière du porche actuel de la grotte, que nous appellerons niveau 9. Cet ensemble a livré des éléments rapportables à la méthode Discoïde, alors que d'autres sont issus d'un système de débitage Levallois ;

- un imposant ensemble de vestiges lithiques et osseux, situé dans la pente en avant du gisement (zone 2 supra) qui, lui-même, peut être divisé en deux :

- au sommet, de rares pièces d'allure Paléolithique supérieur voisinent avec des objets d'allure Paléolithique moyen. Ces artefacts sont emballés dans un sédiment brun terreux. II n'est pas exclu qu'ils représentent en partie les déblais des fouilles Labrot, redistribués dans la pente (Bordes 2002 op. cit.). 


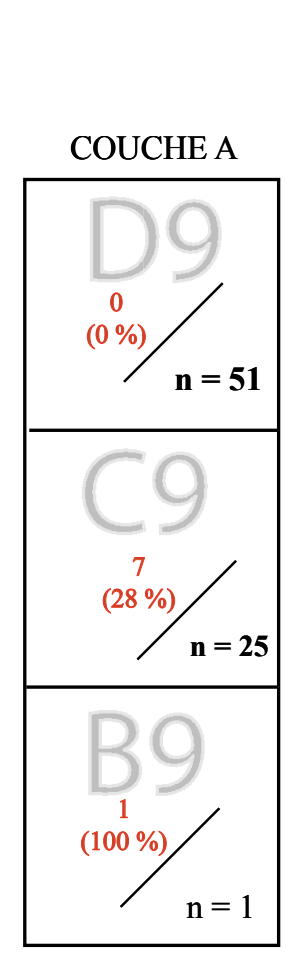

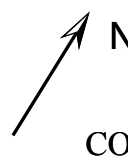

COUCHE B

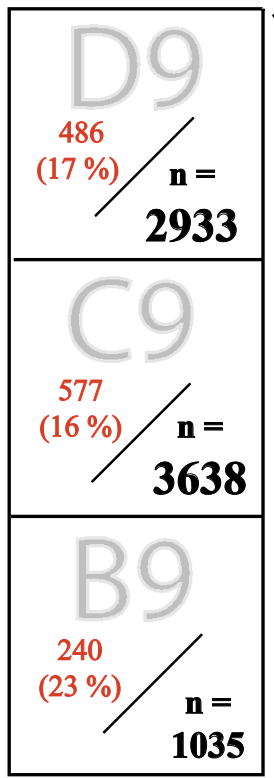

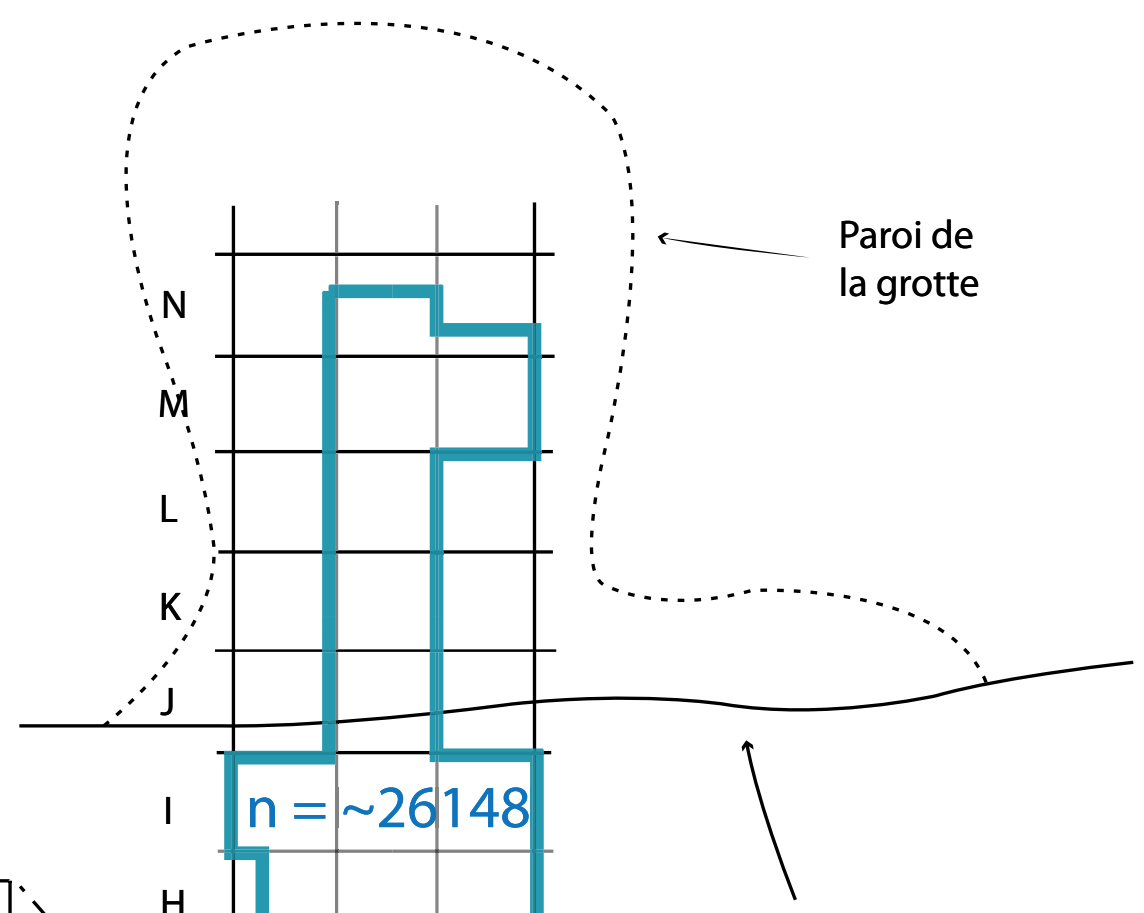

Limite de la falaise

Figure 3 - Roc de Combe (Lot) - Vue en plan du gisement. En bleu : secteur fouillé de la grotte et nombre de pièces moustériennes total estimé. En gris : carrés étudiés dans ce travail. Les nuances de gris (du plus foncé au plus clair) sont fonction du nombre de pièces. À gauche : ensemble du matériel échantillonné décompté par carré et par couche. En rouge : nombre et proportion des pièces cotées par carré. D’après Bordes 2002, modifié.

Figure 3 - Roc de Combe (Lot) - Plan view of the site. In blue: excavated area of the cave and estimated total number of pieces. In gray: square studied in this work. Shades of gray (darker to lighter) depend to the number of pieces. Left: all the sampled material and counted per square and layer. In red: number and proportion of coordinated per square pieces. After Bordes 2002, modified. 
- pour le reste des dépôts, on rencontre une industrie lithique homogène, définie par un concept de débitage Discoïde, dont l'outillage est dominé par les outils denticulés et les encoches. Le sédiment emballant cet ensemble est jaune, granuleux ou terreux.

Une stratigraphie sommaire de cet ensemble avait été établie lors de la fouille, mais non reprise ici pour plusieurs raisons : végétalisation postérieure ; matériel lithique homogène ; délimitation incertaine d'après les fouilleurs eux-mêmes, notamment en raison d'un fort encroûtement carbonaté ; fouille des déblais Labrot probablement assimilés à une séquence archéologique. L'hypothèse privilégiée expliquant la formation de cet ensemble serait une redistribution post-dépostionnelle (Bordes 2002). À ce jour, la faune de cet ensemble moustérien demeure inédite. Rien ne permet a priori de préciser le nombre d'occupations que cet ensemble recouvre.

\section{3 - La série F. Bordes du MNP et son échantillonnage}

Nous avons pu estimer la série lithique moustérienne actuellement déposée au musée national de Préhistoire (MNP, Les Eyzies-de-Tayac) à plus de 26000 artefacts. Par contrainte de temps imparti pour un travail universitaire ${ }^{5}$, mais aussi pour la raison que l'on vient d'avancer, un échantillonnage fut réalisé, portant sur trois mètres carrés considérés comme représentatifs en termes de topographie et de densité archéologique, totalisant au moins 7610 pièces, soit $43 \%$ du matériel lithique de la zone 2 et un peu moins du tiers de la collection moustérienne dans son ensemble (29\%) (fig. 3 et tabl. 1).

\section{4 - Méthodologie, cadre archéopétrographique}

\subsection{1 - Concepts et vocabulaire typo-technologiques}

L'étude de la série a compris une phase de tri selon les différentes catégories de supports reconnus d'après la littérature francophone consacrée aux séries du Paléolithique moyen (Geneste 1985 ; Turq 1992, 2000 ; Boëda 1993 ; Jaubert 1994 ; Locht et Swinnen 1994 ; Mourre 2003 ; Slimak 2003 ; Locht 2004 ; Faivre 2008). Un corpus de données métriques a été établi : seules ont été mesurées les longueur, largeur et parfois épaisseur, dans leur axe de débitage, des pièces présentant un intérêt pour l'étude (nucléus, supports retouchés, produits spécifiques du débitage Discoïde). L'analyse des nucléus a été effectuée par une série de descripteurs reconnus, que nous détaillerons. L'observation des retouches est fondée sur les travaux de Inizan, Reduron-Bollinger, Roche 1995 et Thiébaut 2005 pour les outils denticulés.

Notre inventaire lithologique est largement inspiré des recherches menées antérieurement pour l'Aurignacien ancien du même site (Bordes 2002) ainsi que des divers travaux princeps, toutes périodes confondues (Morala 1984 ; Geneste 1985 ; Séronie-Vivien et Séronie-Vivien 1987 ; Astruc 1990 ; Turq, Antignac, Roussel 1999 ; Turq 2000) ou plus récents (Faivre 2008 ; Turq 2000 ; Turq et Morala 2013). Les diagnostics pétrographiques ont été effectués grâce au concours d'A. Morala et A. Turq (MNPPACEA) que nous remercions.

\section{3 - L'industrie lithique du Moustérien}

\section{1 - Matières premières}

La dimension moyenne des objets abandonnés est similaire d'une matière première à l'autre. Les données pondérales non prises en compte - auraient été biaisées par le fait que la patine a pu modifier la masse initiale des objets. Les données chiffrées discutées infra sont les nombres de pièces pour chaque catégorie. Les Moustériens de Roc de Combe ont principalement exploité la calcédoine tertiaire, qui constitue $79 \%$ de la série décomptée, puis des petits blocs de silex du Sénonien (gris/noir et blond), soit $10 \%$ de l'ensemble (tabl. 1). On rencontre ensuite les quartz et métaquartzites $(8 \%)$. Divers autres types de silex sont

\begin{tabular}{|c|c|c|c|c|c|c|c|c|c|c|c|c|c|}
\hline \multirow{2}{*}{\multicolumn{2}{|c|}{ Catégories technologiques }} & \multicolumn{3}{|c|}{ Calcédoine tertiaire } & \multicolumn{3}{|c|}{ Silex sénonien } & \multicolumn{3}{|c|}{ Autres silex } & \multicolumn{3}{|c|}{ Quartzites } \\
\hline & & Effectif & $\%$ & $\begin{array}{c}\text { \% par rapport à } \\
\text { la série totale }\end{array}$ & Effectif & $\%$ & $\begin{array}{c}\% \text { par rapport à } \\
\text { la série totale }\end{array}$ & Effectif & $\%$ & $\begin{array}{l}\text { \% par rapport à } \\
\text { la série totale }\end{array}$ & Effectif & $\%$ & $\begin{array}{l}\text { \% par rapport à } \\
\text { la série totale }\end{array}$ \\
\hline \multirow{3}{*}{ Éclats à surface naturelle } & Entames & 162 & $2,6 \%$ & $2,1 \%$ & 44 & $5,8 \%$ & $0,6 \%$ & 1 & $1 \%$ & $>0,1 \%$ & 8 & $1,3 \%$ & $0,1 \%$ \\
\hline & Éclats corticaux > 50\% & 453 & $7,4 \%$ & $5,8 \%$ & 66 & $8,7 \%$ & $0,8 \%$ & 5 & $5 \%$ & $0,06 \%$ & 82 & $12,9 \%$ & $1,1 \%$ \\
\hline & Éclats à cortex résiduel $<50 \%$ & 1092 & $17,9 \%$ & $14,1 \%$ & 154 & $20,4 \%$ & $2,0 \%$ & 16 & $15 \%$ & $0,21 \%$ & 189 & $29,8 \%$ & $2,5 \%$ \\
\hline Éclats indifférenciés & Éclats sans cortex & 926 & $15,1 \%$ & $11,9 \%$ & 101 & $13,4 \%$ & $1,3 \%$ & 26 & $24 \%$ & $0,33 \%$ & 96 & $15,1 \%$ & $1,3 \%$ \\
\hline \multirow{3}{*}{$\begin{array}{c}\text { Produits prédéterminants et } \\
\text { prédéterminés }\end{array}$} & Éclats centrés & 446 & $7,3 \%$ & $5,7 \%$ & 50 & $6,6 \%$ & $0,6 \%$ & 2 & $2 \%$ & $>0,1 \%$ & 16 & $2,5 \%$ & $0,2 \%$ \\
\hline & Éclats débordants & 330 & $5,4 \%$ & $4,2 \%$ & 41 & $5,4 \%$ & $0,5 \%$ & 5 & $5 \%$ & $0,06 \%$ & 17 & $2,7 \%$ & $0,2 \%$ \\
\hline & Pointes pseudo-Levallois & 220 & $3,6 \%$ & $2,8 \%$ & 24 & $3,2 \%$ & $0,3 \%$ & 10 & $9 \%$ & $0,13 \%$ & 14 & $2,2 \%$ & $0,2 \%$ \\
\hline \multicolumn{2}{|c|}{ Éclats Kombewa } & 121 & $2,0 \%$ & $1,6 \%$ & 29 & $3,8 \%$ & $0,4 \%$ & 3 & $3 \%$ & $>0,1 \%$ & 1 & $0,2 \%$ & $>0,1 \%$ \\
\hline \multicolumn{2}{|c|}{ Fragments } & 1508 & $24,7 \%$ & $19,4 \%$ & 136 & $18,0 \%$ & $1,8 \%$ & 29 & $27 \%$ & $0,37 \%$ & 93 & $14,7 \%$ & $1,2 \%$ \\
\hline \multicolumn{2}{|c|}{ Éclats de retouche } & 298 & $4,9 \%$ & $3,8 \%$ & 60 & $7,9 \%$ & $0,8 \%$ & 0 & & & 0 & & \\
\hline \multicolumn{2}{|c|}{ Nucléus } & 185 & $3,0 \%$ & $2,4 \%$ & 36 & $4,8 \%$ & $0,5 \%$ & 4 & $4 \%$ & $0,05 \%$ & 33 & $5,2 \%$ & $0,4 \%$ \\
\hline \multicolumn{2}{|c|}{ Cassons } & 363 & $5,9 \%$ & $4,7 \%$ & 14 & $1,9 \%$ & $>0,1 \%$ & 3 & $3 \%$ & $>0,1 \%$ & 62 & $9,8 \%$ & $0,8 \%$ \\
\hline \multicolumn{2}{|c|}{ Indéterminés } & 10 & $0,2 \%$ & $0,1 \%$ & & & & 3 & $3 \%$ & $>0,1 \%$ & 23 & $3,6 \%$ & $0,3 \%$ \\
\hline \multicolumn{2}{|c|}{ Total } & 6114 & $100 \%$ & $78,7 \%$ & 755 & $100 \%$ & $9,7 \%$ & 107 & $100 \%$ & $1,4 \%$ & 634 & $100 \%$ & $8,3 \%$ \\
\hline
\end{tabular}

Tableau 1 - Roc de Combe (Lot) - Décompte technologique des pièces par matière première.

Table 1 - Roc de Combe (Lot) - Technological counting per lithic raw material.

(5) Master 2 université Bordeaux 1 : Lorenzo Martinez 2012. 
présents en proportions infimes : jaspéroïde de l'Infralias, silex Maastrichtien dit « Bergeracois », Turonien inférieur dit « Fumélois », silex du Portlandien ou silex de Missère (Lot), silex du Bathonien et enfin silex coniacien inférieur dit «Gavaudun » (présence probable). II conviendrait d'ailleurs de confirmer ces diagnostics par des analyses pétrographiques plus précises. Quelques autres types de matériaux non crypto-cristallins sont présents de façon anecdotique : basalte, grès ferrugineux et grès. Compte-tenu des sources d'affleurement potentielles de ces matériaux, l'approvisionnement est donc très majoritairement local (sensu Geneste 1985), la zone d'acquisition de $99 \%$ des matériaux recensés dans la série est en effet très probablement circonscrite dans un rayon inférieur à $10 \mathrm{~km}$, englobant les vallées de la Marcillande, de la Germaine et une portion de la vallée de la Dordogne. En cela, Roc de Combe présente de nombreux points communs avec le bilan de la Grotte Vaufrey, voisine d'une quinzaine de kilomètres (Geneste 1988).

Ce spectre local concerne le silex tertiaire lacustre et le jaspéroïde (présent dans les alluvions de la Dordogne), le Sénonien gris/noir, le Portlandien et enfin les blocs alluviaux de quartzites ou de basalte ainsi que les grès des plateaux. Cependant, la présence de silex relevant d'une zone régionale voisine (15-35 km) est également attestée, à savoir quelques variétés de silex jurassiques. Enfin, les Moustériens de Roc de Combe ont compté comme lieux d'approvisionnement ou d'échanges des sources plus éloignées (de 40 à $70 \mathrm{~km}$ ) représentés par les silex du Turonien inférieur, dit « Fumélois », du Coniacien inférieur (Gavaudun) - ce dernier sans attestation certaine - et enfin du Maastrichtien de type «Bergeracois ». Des précisions pourraient être apportées par une caractérisation pétrographique plus fine de certaines pièces. Toutefois, ces premières estimations constituent déjà une sérieuse base documentaire, mettant en évidence quelques traits majeurs de cette série :

- exploitation très majoritaire de l'environnement local, privilégiant des matériaux de qualité relativement médiocre, irrégulière, mais au tranchant somme toute efficace ;

- acquisition quantitativement décroissante selon les catégories d'éloignement des gîtes de matières premières lithiques ;

- prélèvement de blocs pour une utilisation autre que la taille (grès, granite) ;

- présence de quelques matières lointaines, mais en quantités anecdotiques : silex rares et remarquables, à savoir le Fumélois, ce dernier bien attesté, au contraire du Gavaudun qui mériterait une attention toute particulière même s'il n'est représenté que par une seule pièce ; enfin le «Bergeracois » (Maastrichtien, cf. infra).

\section{2 - Analyse techno-économique}

\subsection{1 - Caractéristiques technologiques générales}

L'industrie se démarque par une extrême homogénéité des méthodes employées dans l'exploitation des différents supports, sans différenciation des matières premières. Ainsi, la quasi-totalité de la série peut être rapportée à une seule et même méthode, le débitage Discoïde (Turq 1992 ; Boëda 1993 ; Jaubert 1993, 1994 ; Locht et Swinnen 1994 ; Slimak 1999, 2003 ; Brenet et Folgado 2003 ; Mourre 2003 ; Peresani (dir.) 2003).

Les talons des éclats, majoritairement lisses sont généralement larges et épais, les bulbes et points d'impacts très marqués, ce qui va dans le sens de l'emploi privilégié, voire exclusif, de la technique de percussion directe au percuteur dur selon un geste rentrant, ce que corrélerait la présence de galets de quartzite $(n=14$, soit 2,2 \%), débités ou non, portant des impacts de percussion sur le néocortex, et majoritairement fracturés.

Un peu moins de $10 \%$ des supports produits sont repris par des enlèvements eux aussi effectués à la pierre selon un geste rentrant, de façon alternante, aboutissant à des encoches et denticulés.

\subsection{2 - Méthodes de débitage et production}

\section{Supports de débitage}

Le débitage s'est effectué aux dépens de blocs $(n=80$, soit $31 \%$ des nucléus), d'éclats ( $n=137$, soit $53 \%$ ) ou enfin aux dépens de galets ( $n=32$ soit $40 \%$ ). Nous avons distingué les nucléus en fonction du nombre de surfaces débitées, puis des modalités mises en œuvre pour leur exploitation, qui peut-être réduite à un ou deux éclats extraits, ou plus complexe. En l'absence de remontage, nos observations sont fondées sur la morphologie finale des nucléus lesquels permettent de visualiser la dernière séquence effectuée.

\section{Les nucléus}

Deux types principaux de nucléus en silex se dégagent :

- d'une part, une production à partir de nucléus sur éclats corticaux de dimensions moyennes selon des modalités unipolaire, bipolaire et convergente aux dépens d'une ou de deux surfaces, avec un fort degré d'aplatissement ( $\mathrm{n}=$ 85 , soit $32 \%$ des nucléus) (fig. $4, n^{\circ} 2$ et 4 ) ;

- d'autre part, des nucléus sur blocs ont été entièrement décortiqués et débités selon une modalité centripète $(n=43$ soit $16 \%$ ) ; ils sont très réduits et bien entretenus, avec une propension à devenir globuleux (fig. $4, \mathrm{n}^{\circ} 1$ et 3 ).

Les nucléus en silex sénonien présentent une exploitation bien plus poussée que ceux en calcédoine tertiaire, avec des modules plus réduits et une productivité plus importante. Les nucléus en métaquartzite se caractérisent quant à eux par une exploitation essentiellement unifaciale, sur de grands galets ; ils sont typiques des nucléus discoïdes en quartz et quartzite (Jaubert et al. 1990 ; Jaubert 1994 ; Mourre 1996 ; Jaubert et Mourre 1996), avec une surface de débitage opposée à une réserve néocorticale, présentant une section pyramidale. Ils sont caractéristiques des séries discoïdes en quartz et quartzites déjà documentées dans la région (e.g. La Borde : Jaubert et al. 1990 ; Mauran : Jaubert 1994 ; couche G7 des Fieux : Faivre 2004 ; Coudoulous 1 c4 : Mourre 1994 ; Les Bosses : Jarry et al. 2007 ; Faivre et al. 2013) (fig. 5). 


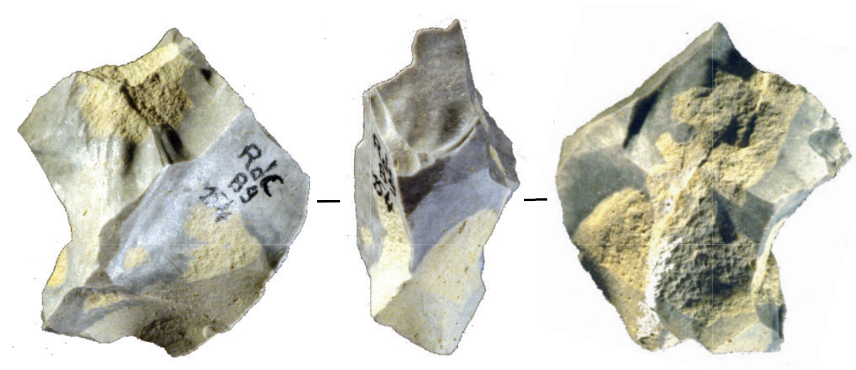

1
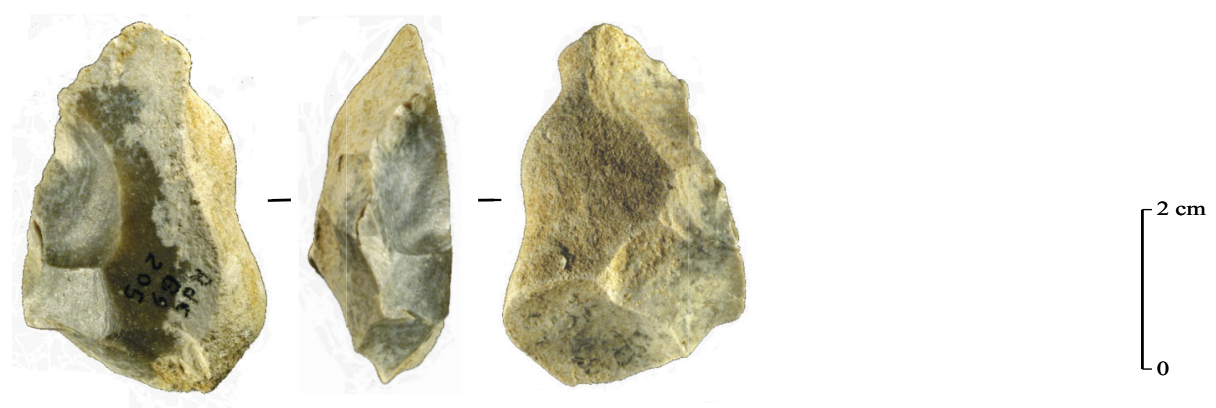

2

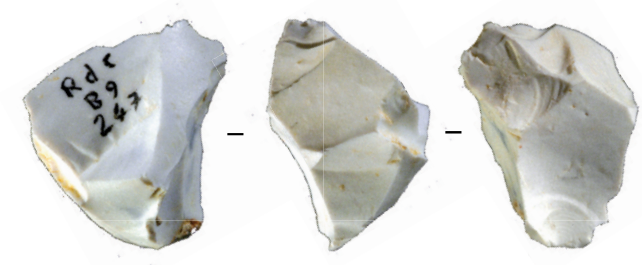

3
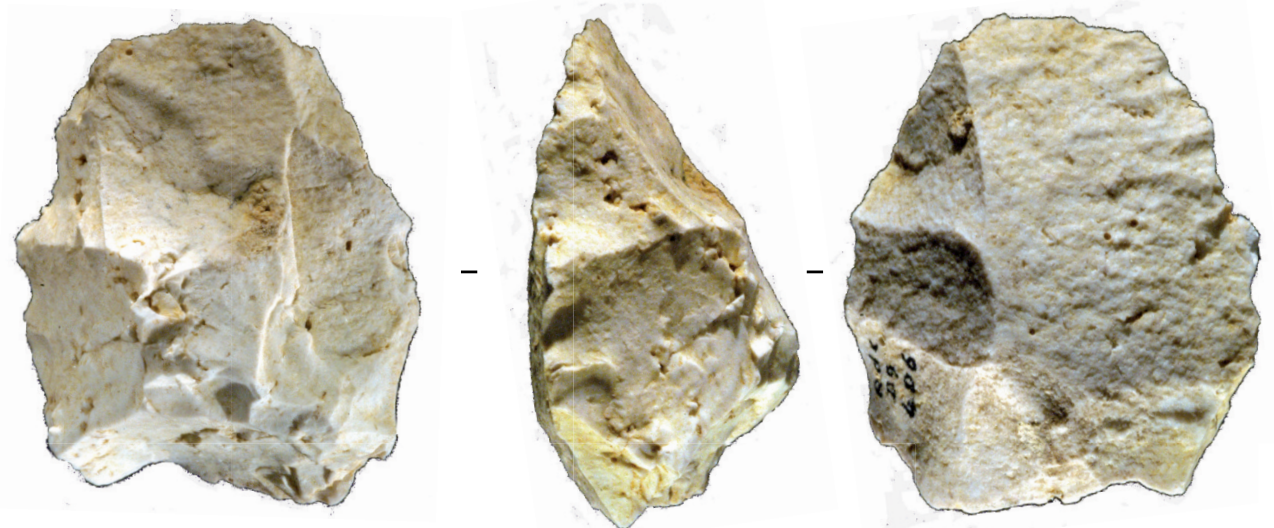

4

Figure 4 - Roc de Combe (Lot), Moustérien Discoïde à denticulés. Nucléus discoïdes sur bloc et sur éclat. 1 : nucléus en silex sénonien sur bloc de débitage centripète typiquement Discoïde. 2 : nucléus en silex sénonien sur éclat cortical bifacial de modalité unipolaire. 3 : nucléus en calcédoine tertiaire sur bloc de section bipyramidale. 4 : nucléus en calcédoine tertiaire sur éclat de modalité centripète. () MNP Les Eyzies - Dist. RMN- cliché Ph. Jugie.

Figure 4 - Roc de Combe (Lot), Moustérien Discoïde à denticulés. Cores on block and flake-core. 1: Discoid senonian flint core on block reduced following a centripetal modality. 2: Senonian flint cortical flake-core reduced following an unipolar modality. 3: bipyramidal core on block in "tertiaire". 4: flake-core reduced following a centripetal modality in "tertiaire". Credits : MNP Les Eyzies - Dist. RMN- photo Ph. Jugie. 

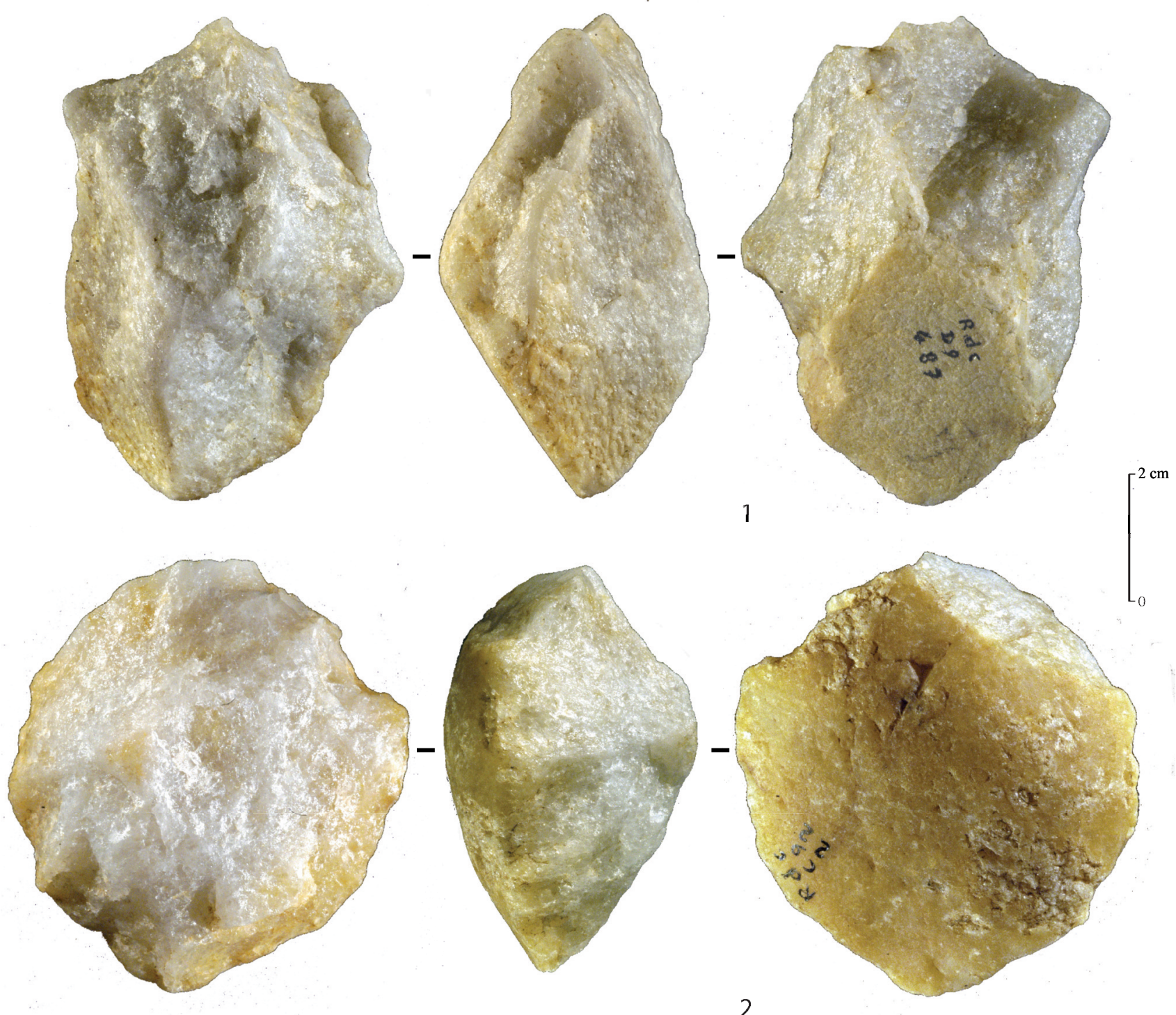

Figure 5 - Roc de Combe (Lot), Moustérien Discoïde à denticulés. Nucléus en quartzite. 1 : nucléus bifacial discoïde. 2 : nucléus unifacial discoïde. () MNP Les Eyzies - Dist. RMN- cliché Ph. Jugie.

Figure 5 - Roc de Combe (Lot), Moustérien Discoïde à denticulés. Quartzite cores. 1: bifacial Discoid core. 2: unifacial Discoid core. Credits: MNP Les Eyzies - Dist. RMN- photo Ph. Jugie.

\section{Les produits}

Les produits obtenus sont principalement des éclats indifférenciés, corticaux ou non, ainsi que des éclats typiques du débitage Discoïde, tels que pointes pseudoLevallois, éclats débordants et éclats centrés (Bordes 1961; Boëda, Geneste, Meignen 1990 ; Boëda 1993, 1995 ; Meignen 1993 ; Bourguignon et Turq 2003 ; Mourre 2003 ; Slimak 2003 ; Faivre 2008) (tabl. 1 et fig. 6 et 7). Nous avons pu distinguer d'une part, des éclats centrés longs et des éclats débordants envahissants qui permettent d'aplanir les convexités et, d'autre part, des pointes pseudo-Levallois et des éclats centrés courts dont le détachement rehausse les convexités. Le débitage s'est opéré selon une angulation sécante et une direction centripète (éclats centrés) ou « cordale » (éclats débordants lato sensu). Une certaine standardisation est manifeste à travers l'obtention de supports asymétriques, qu'ils soient à dos opposé à un tranchant ou à talon épais avec un tranchant localisé sur le pourtour. Ces éclats se regroupent sous l'appellation " éclats prédéterminants et prédéterminés 》 (Boëda 1993) car ils endossent le rôle ambivalent de finalité de production et ou d'auto-entretien de préparation du nucléus. Le faible taux de ce type de produits en comparaison avec d'autres séries pourrait s'expliquer par l'exportation de ces derniers (tabl. 2 et fig. 8). Les effectifs et le recoupement avec d'autres séries régionales montrent que ces produits signent l'intentionnalité du débitage. 

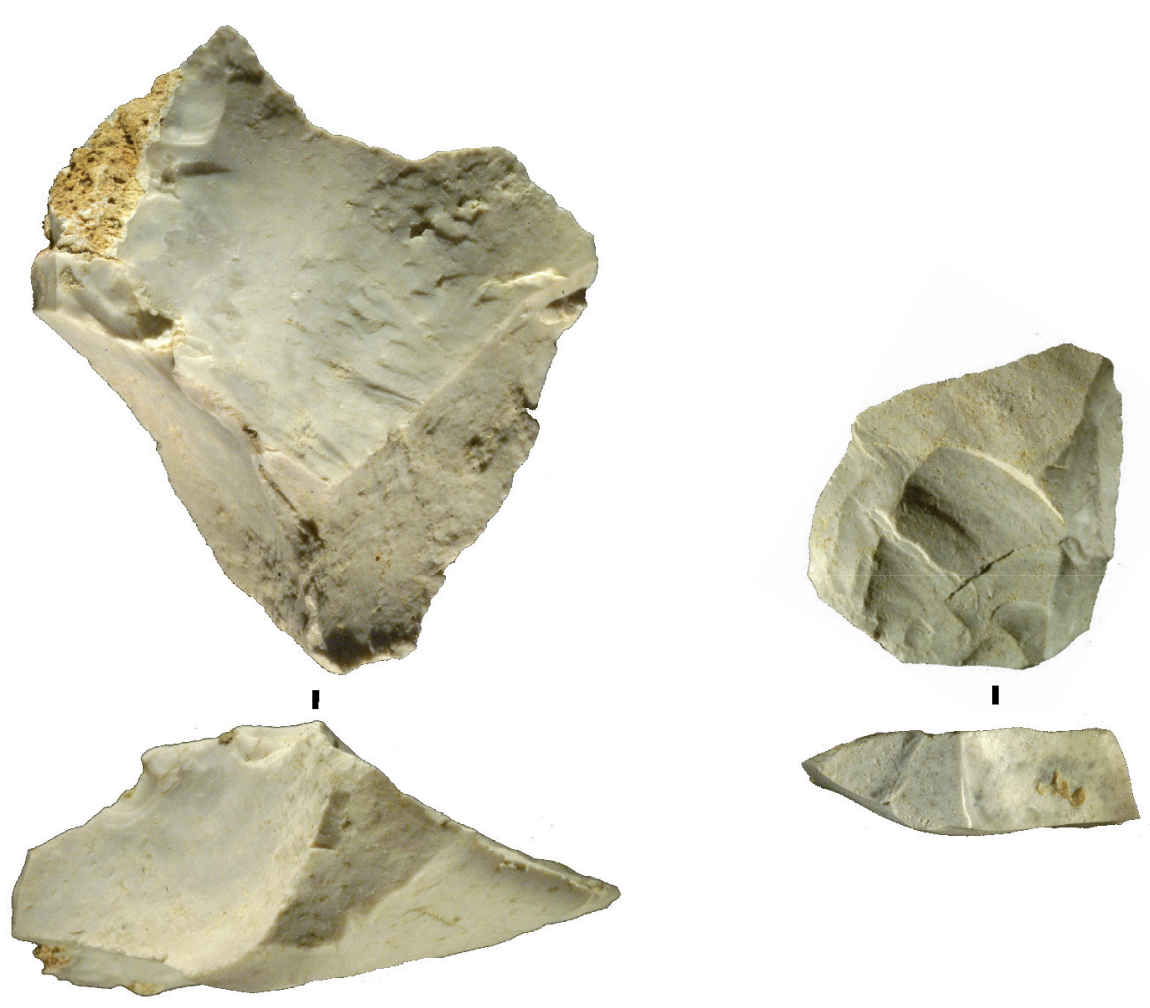

1
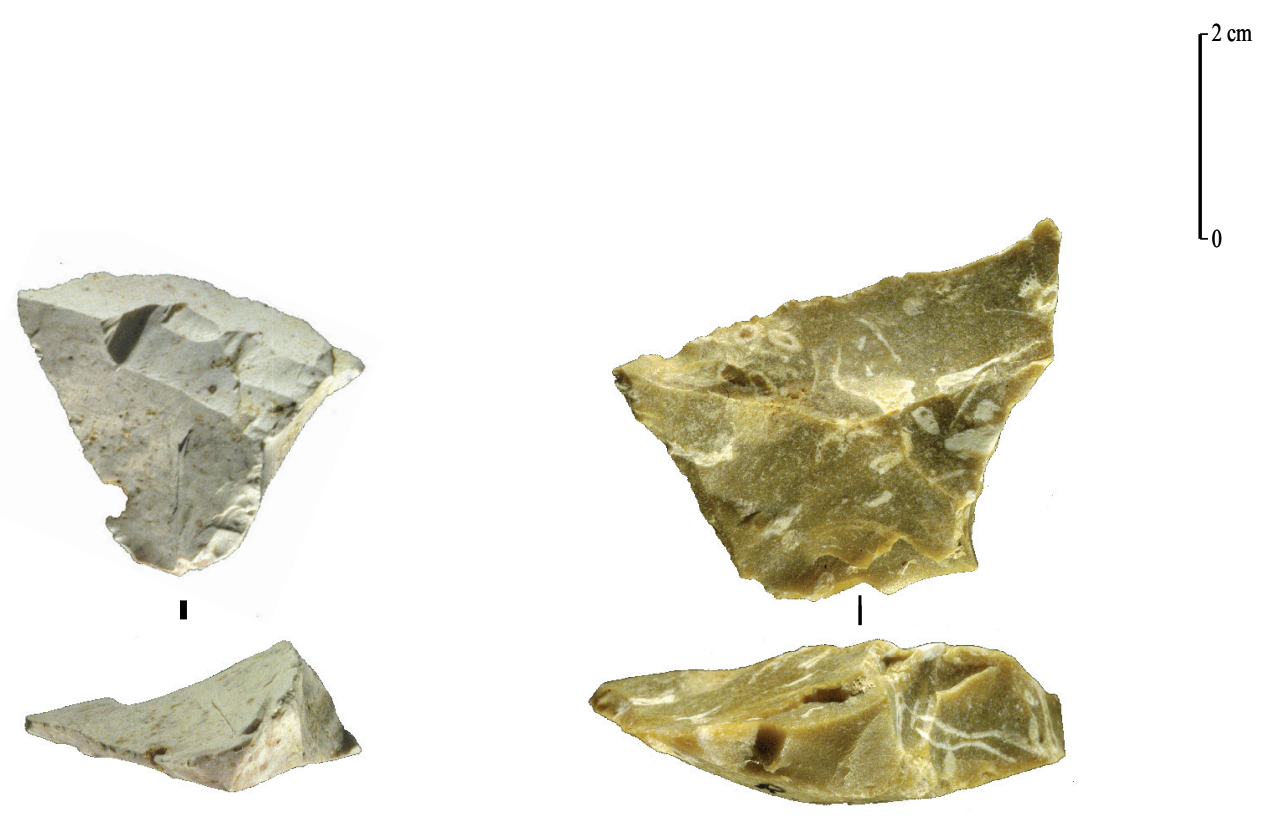

$\left[_{0}^{2 \mathrm{~cm}}\right.$

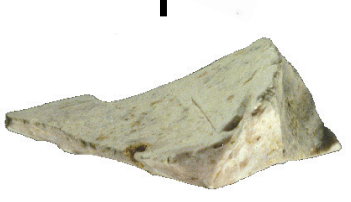

3

4

Figure 6 - Roc de Combe (Lot), Moustérien Discoïde à denticulés. 1 : pointe pseudo-Levallois typique en calcédoine tertiaire. 2 et 3 : pointes pseudo-Levallois atypiques en calcédoine tertiaire. 4 : pointe pseudo-Levallois typique en silex sénonien. (C) MNP Les Eyzies - Dist.RMN- cliché Ph. Jugie.

Figure 6 - Roc de Combe (Lot), Denticulate-Discoïd Mousterian. 1: Typical pseudo-Levallois point in Cenozoic calcedony. 2 and 3: atypical pseudo-Levallois point in Cenozoic calcedony. 4: Senonian flint typical pseudo-Levallois point. Credits: MNP Les Eyzies - Dist. RMN- photo Ph. Jugie. 


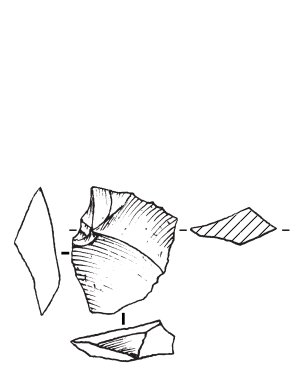

1

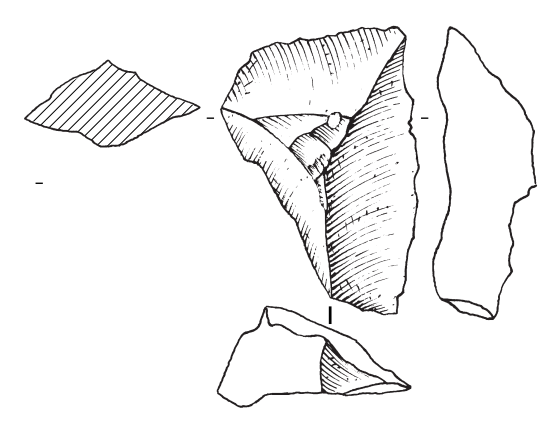

2
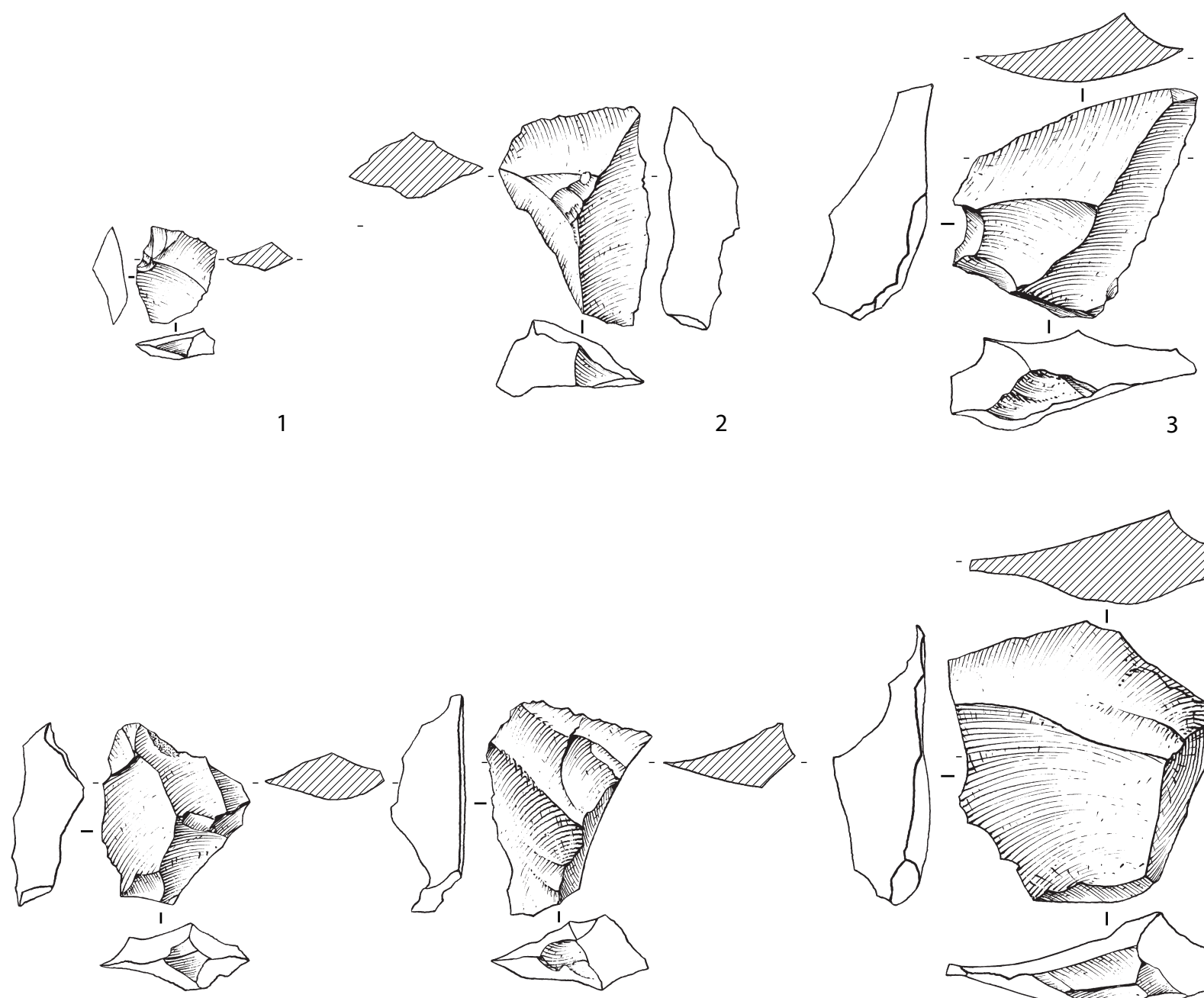

4

5

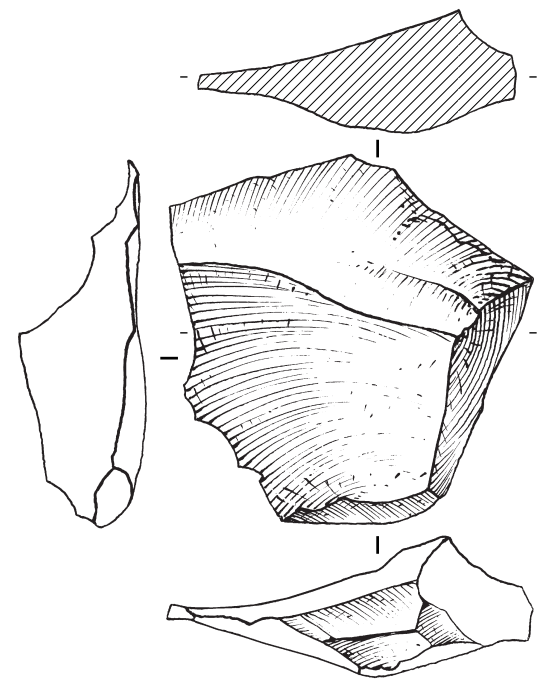

6
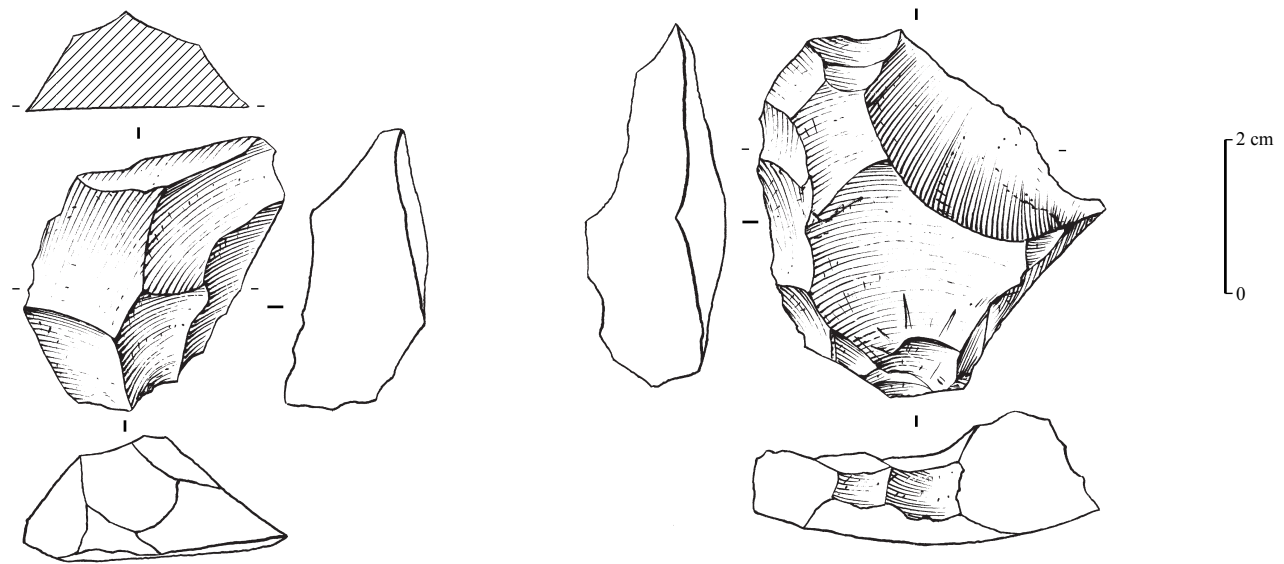

7

8

Figure 7 - Roc de Combe (Lot), Calcédoine tertiaire. 1, 2, 3, et 6 : pointes pseudo-Levallois typiques. 4 et 5 : pointes pseudo-Levallois atypiques. 7 et 8 : éclats débordants de modalité centripète. Dessins : G. Devilder.

Figure 7 - Roc de Combe (Lot), Flint industry in Cenozoic calcedony. 1, 2, 3 and 6: pseudo-Levallois points. 4 and 5: atypical pseudoLevallois points. 7 and 8: centripetal debordant flakes. Drawings: G. Devilder. 


\begin{tabular}{|c|c|c|c|c|c|c|c|c|}
\hline Sites & $\begin{array}{l}\text { Pointes } \\
\text { pseudo- } \\
\text { Levallois }\end{array}$ & $\%$ & $\begin{array}{c}\text { Éclats } \\
\text { débordants }\end{array}$ & $\%$ & $\begin{array}{l}\text { Éclats } \\
\text { centrés }\end{array}$ & $\%$ & $\begin{array}{c}\text { Total produits } \\
\text { (MP } \\
\text { dominante) }\end{array}$ & Bibliographie \\
\hline $\begin{array}{c}\text { SAINT } \\
\text { CÉSAIRE }\end{array}$ & 299 & $7 \%$ & 465 & $11 \%$ & 114 & $3 \%$ & 4170 & Thiébaut 2005 \\
\hline MAURAN & 56 & $7 \%$ & - & - & - & - & 763 & Jaubert 1994 \\
\hline $\begin{array}{c}\text { BEAUVAIS } \\
\text { NIV.1 }\end{array}$ & & & & $7 \%$ & 110 & $6 \%$ & 1780 & Locht 2004 \\
\hline $\begin{array}{c}\text { BEAUVAIS } \\
\text { NIV. } 2\end{array}$ & 348 & $4 \%$ & 208 & $2 \%$ & 331 & $3 \%$ & 9879 & Locht 2004 \\
\hline $\begin{array}{l}\text { CHAMPS DE } \\
\text { BOSSUET }\end{array}$ & 926 & $8 \%$ & 1841 & $16 \%$ & - & - & 11359 & $\begin{array}{c}\text { Bourguignon Turq } \\
2003\end{array}$ \\
\hline $\begin{array}{l}\text { COMBE } \\
\text { GRENAL C } \\
11\end{array}$ & & & & $8 \%$ & - & - & 1499 & Thiébaut 2005 \\
\hline $\begin{array}{l}\text { COMBE } \\
\text { GRENAL C } \\
14\end{array}$ & 174 & $6 \%$ & 543 & $19 \%$ & - & - & 2884 & $\begin{array}{c}\text { Bourguignon Turq } \\
2003\end{array}$ \\
\hline $\begin{array}{c}\text { COMBE } \\
\text { GRENAL C } \\
12\end{array}$ & 148 & $18 \%$ & 143 & $17 \%$ & 98 & $12 \%$ & 838 & Faivre 2008 \\
\hline $\begin{array}{l}\text { LES FIEUX } \\
\text { G7 }\end{array}$ & 60 & $10 \%$ & 45 & $8 \%$ & 47 & $8 \%$ & 585 & Faivre 2002 \\
\hline LES FIEUX I J & 256 & $28 \%$ & 112 & $12 \%$ & 148 & $16 \%$ & 900 & Faivre 2008 \\
\hline $\begin{array}{c}\text { LES } \\
\text { ROCHERS J }\end{array}$ & 17 & $7 \%$ & 5 & $2 \%$ & 11 & $4 \%$ & 258 & Asselin 2005 \\
\hline $\begin{array}{c}\text { LES } \\
\text { ROCHERS N }\end{array}$ & 28 & $27 \%$ & 26 & $25 \%$ & 37 & $36 \%$ & 103 & Asselin 2006 \\
\hline
\end{tabular}

Tableau 2 -

Comparaison des proportions des éclats prédéterminants et prédéterminés par rapport aux produits des sites intégrés dans le Discoïde "stricto sensu " (si données disponibles).

Table 2 -

Comparison of proportions of predetermining and predetermined flakes with respect to blanks included in the Discoid «stricto sensu» sites (if available data).

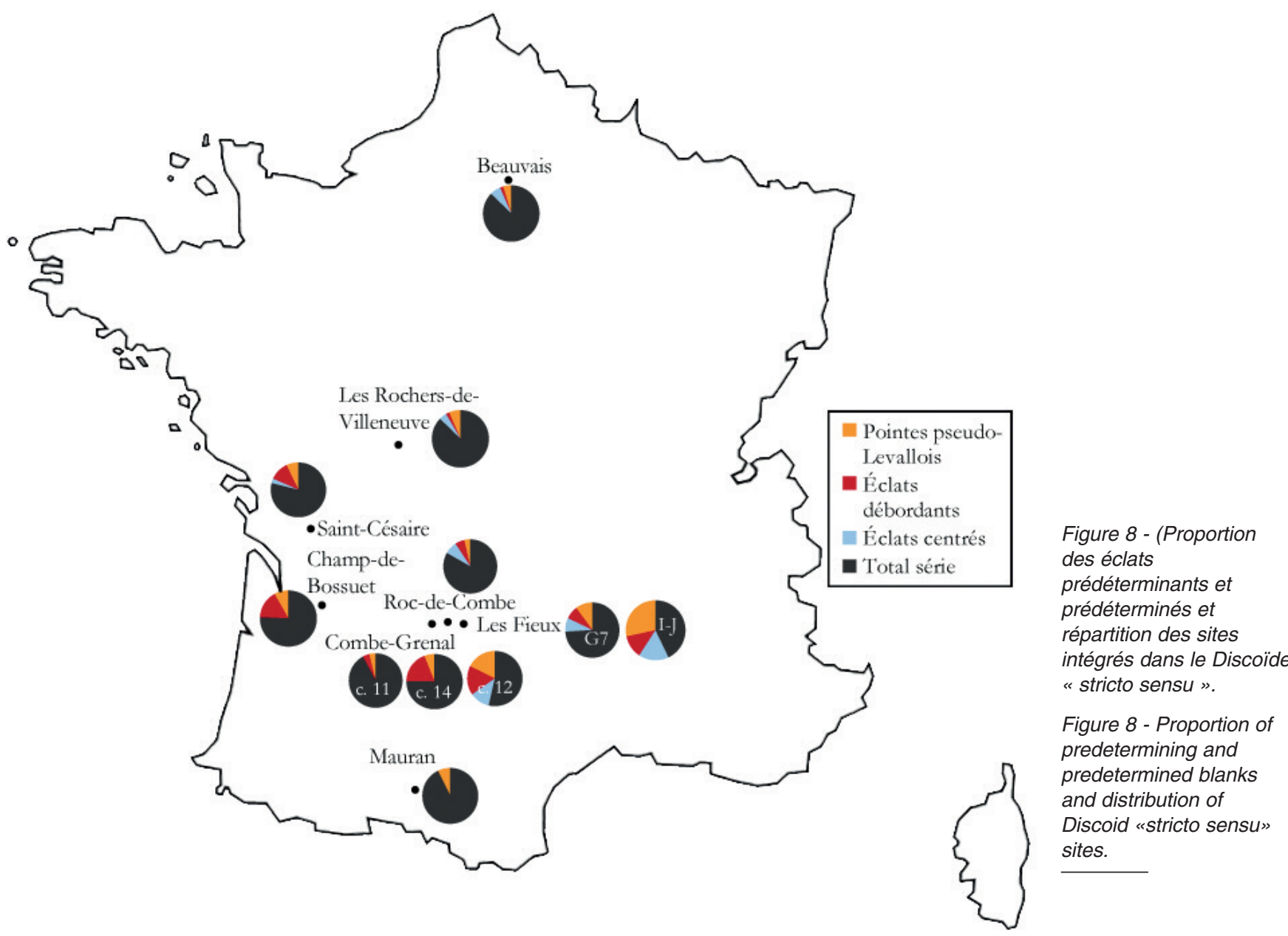


Cette hypothèse est renforcée par l'analyse du dernier négatif d'enlèvement sur les nucléus qui montre que les éclats indifférenciés non corticaux et les éclats prédéterminants et prédéterminés se retrouvent en proportions quasiment équivalentes (respectivement $44 \%$ et $56 \%$ ). Toutefois, le silex sénonien ne présente pas les mêmes résultats avec une plus forte prédominance des éclats prédéterminants et prédéterminés.

\subsection{3 - Gestion des produits de débitage et composition typologique de l'outillage retouché}

\section{Supports transformés}

Le taux de transformation des produits est plutôt faible $(6,78 \%$ des produits, toutes catégories technologiques confondues, hormis les cassons) (fig. 9). Cependant, ce taux masque d'importantes différenciations pour chaque matière première (tabl. 3). Ainsi, les supports retouchés en calcédoine tertiaire ne représentent que $7,1 \%$ au sein de ce matériau, tandis que les outils retouchés constituent $16,9 \%$ pour les supports en silex sénonien. Comme souvent, les quartzites/métaquartzites possèdent le plus faible taux de retouche $(2,3 \%)$. Pour les autres types de silex, 15 pièces sont retouchées, soit une proportion moyenne $(7,4 \%)$.

Les différences résident également dans le choix des supports transformés (tabl. 3) : pour la calcédoine tertiaire, la retouche concerne majoritairement les éclats corticaux $(22,9 \%$ sur la totalité des pièces retouchées) et les fragments d'éclats (19\%), mais en proportion relative, ce sont les éclats débordants lato sensu $(26,8 \%)$ et les éclats de type Kombewa (9,9\%) qui montrent le plus fort taux de retouche. Généralement, les supports retouchés présentent de plus faibles dimensions que ceux restés bruts.

Parmi les silex sénoniens, les supports quantitativement privilégiés pour être retouchés ont clairement été les éclats à cortex résiduel, lesquels dominent les effectifs $(40,4 \%)$. Les éclats corticaux (cortex $<50 \%$ et $>50 \%$ ) et les éclats de type Kombewa ont été préférentiellement transformés au sein de chaque catégorie. Les éclats prédéterminants et prédéterminés présentent des taux de retouche moins élevés. Au sein de chaque catégorie, ce sont les plus grands supports qui ont été sélectionnés, exception faite des éclats largement corticaux, des pointes pseudoLevallois et des fragments d'éclats.

Les supports retouchés en quartzite sont exclusivement des éclats à surface non néocorticale ou comportant un dos naturel dont un bord latéral a été préférentiellement transformé.

\section{Typologie}

Les denticulés constituent de loin la catégorie la plus importante $(n=257)$, soit $49,8 \%$ des supports retouchés (tabl. 4 et fig. $10, \mathrm{n}^{\circ} 1,2,3,4,5$ et 8 ) tous matériaux confondus, suivis par les encoches $(n=102)$ soit $19,7 \%$. Les supports en silex sénonien ont été le plus investis par la retouche denticulée : $57 \%$ contre $45 \%$ pour les éclats en Tertiaire et $40 \%$ pour les quartzites. Les microdenticulés (sensu Thiébaut 2005) (fig. 10, $\mathrm{n}^{\circ} 3$ et 5 ) dominent les outils denticulés (69\%), suivis des denticulés à denticulations moyennes (21\%) (fig. 10, $\mathrm{n}^{\circ} 4$ et 8 ) et enfin des macrodenticulés ( $8 \%$ ) (fig. 10, $n^{\circ} 4$ ]. On rencontre souvent des denticulations opposées à une base épaisse ou à un dos (n $=72$, soit $14 \%$ des outils) (fig. $10, n^{\circ} 4,5$ et 8]. Ce bord a pu alors constituer une zone de préhension ou de maintien, opposée à un tranchant actif. Quelques supports ont été investis par une retouche denticulée convergente sur les bords distaux ( $n=11$ soit $2,1 \%$ des outils), les assimilant à des pointes de Tayac. Contrairement aux pièces denticulées, ce sont les macro-encoches qui dominent les outils encochés (jusque $3 \mathrm{~cm}$ d'ouverture) (fig. 10, $\mathrm{n}^{\circ} 7$ ).

Les racloirs sont très minoritaires $(n=24)$, totalisant seulement $4,7 \%$ de l'outillage retouché et présentent en outre des différences importantes en fonction de la matière première. Absents parmi les éclats en quartzites, ils constituent $6 \%$ des supports retouchés sénoniens et $5 \%$ des outils en Tertiaire. Les racloirs ont été majoritairement aménagés sur des pointes pseudo-Levallois $(29,4 \%)$ et des éclats débordants (23,5\%), par des retouches courtes sur le tranchant opposé au dos, avec une prédominance des racloirs simples concaves. Les racloirs en Sénonien sont essentiellement de types simples, droits, convexes ou concaves. D'autres types d'outils retouchés ont été observés, tels que les outils composites (fig. 10, $n^{\circ} 6$ ) ou porteurs de retouches partielles, non classifiables ou atypiques (tabl. 4).

\section{Quelques pièces discordantes}

Dans cette série techno-typologiquement homogène, et de débitage quasi exclusivement Discoïde, dominée par les denticulés et les encoches, cinq pièces $(0,9 \%$ des outils) assez discordantes sont toutefois présentes. Elles s'inscrivent dans la typologie du Paléolithique récent des niveaux sus-jacents au Châtelperronien (cf. discussion in Bordes 2002 - p. 68 et ss.) et se déclinent comme suit : un grattoir, un fragment distal de petite lame, une partie mésiale de pièce à dos évoquant une pointe de Châtelperron, un court fragment proximal de pièce à dos et un éclat lamellaire à pan revers, provenant probablement d'un réaménagement de burin busqué. Ces éléments proviennent de l'extrême sommet de la zone fouillée, dans une zone où, d'après les fouilleurs, les niveaux étaient mal caractérisés, constitués en partie par les déblais effondrés des fouilles Labrot, notamment dans les niveaux châtelperroniens. II est clair qu'il s'agit de migrations verticales.

\section{Éclats de retouche}

La série comporte 355 éclats de silex de petite taille avec un profil mince, longiligne sur la face supérieure et généralement bombé sur la face inférieure (fig. 11). Leur morphologie et leurs dimensions sont à rapprocher des éclats de retouche, dont plusieurs types ont pu être définis à partir d'exemples publiés (Bourguignon 1997 ; Faivre 2008). On note une diversité morpho-technique, 


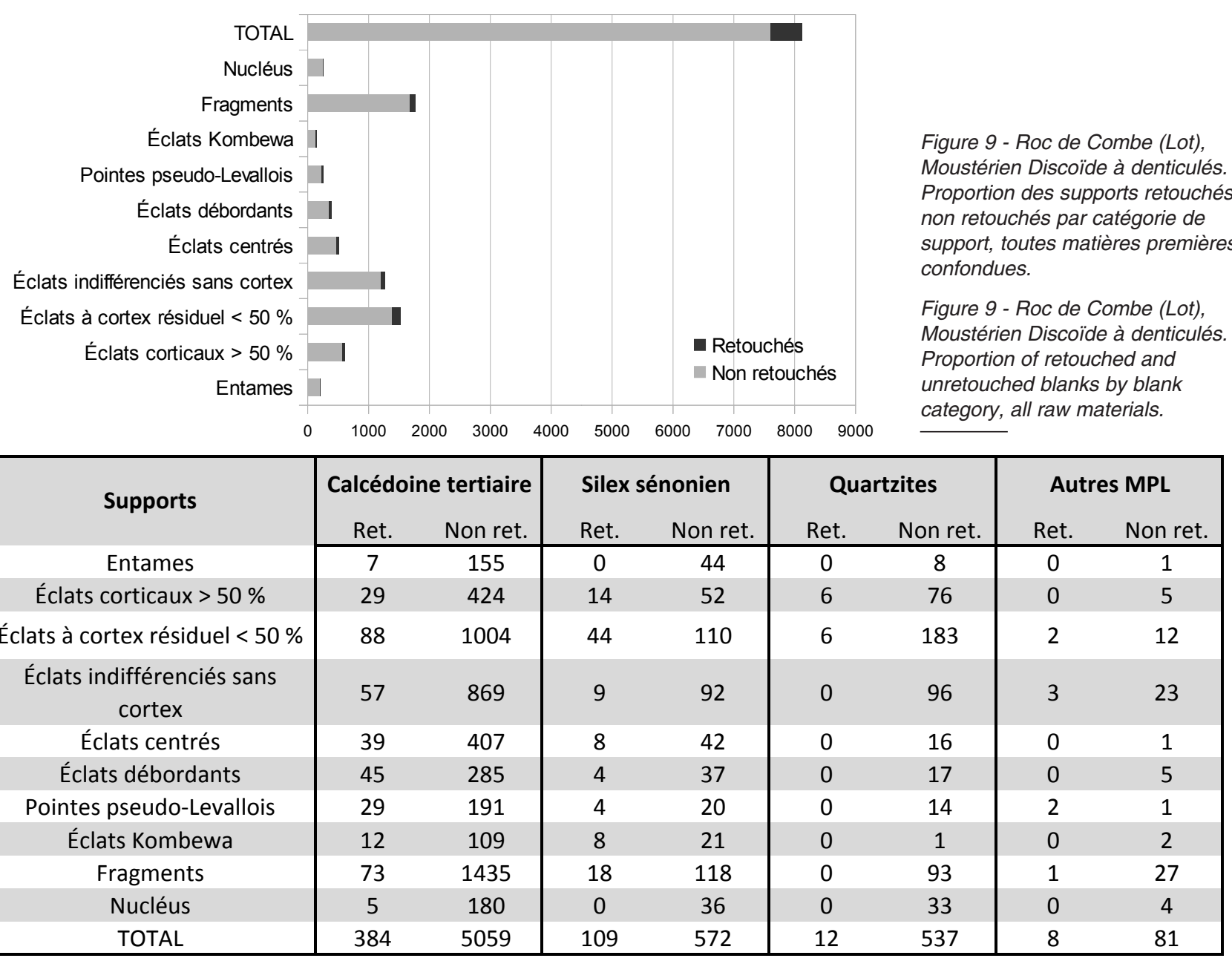

Tableau 3 - Roc de Combe (Lot), Effectifs des supports retouchés et non retouchés par catégorie de support et par matière première.

Table 3 - Roc de Combe (Lot), Number of retouched and unretouched blanks by blank category and lithic raw material.

témoignant de diverses étapes et techniques de transformation des supports. Les éclats d'encoche dominent (38\%) (fig. 11, $\mathrm{n}^{\circ} 1$ et 2 ), immédiatement suivis par les éclats de reprise d'encoche (33\%) (fig. $11 n^{\circ} 3$ ), puis par des éclats de reprise d'outils indéterminés (14\%). Le taux de reprise sur les outils eux-mêmes étant faible, pour ne pas dire anecdotique, on peut signaler ce fort pourcentage de reprise d'outils, plus particulièrement d'encoches. En l'absence de récolte systématique des petits objets, il est cependant difficile d'interpréter ces proportions.

\section{4 - Discussion}

\section{1 - Diagnose générale}

À Roc de Combe, le débitage Discoïde est exclusif, orienté vers l'extraction de supports asymétriques et à bord épais. II se rapproche de séries telles que Beauvais, Oise (Locht 2004), Champs de Bossuet (A89, Gironde) et CombeGrenal, Dordogne, c. 13-15 (Bourguignon et Turq 2003),
Les Fieux, Lot, c. G7 et I-J (Faivre 2008), Mauran, HauteGaronne (Jaubert 1994), Saint-Césaire, CharenteMaritime, Egf-10-11 (Thiébaut, Meignen, Lévêque 2009), La Quina, Charente, c. 5-Gc (Park 2007) ou les Rochers de Villeneuve, Vienne, c.N (Asselin 2005). Cependant, la variabilité technologique est restreinte (les débitages centripète et assimilés prédominent), d'où l'intégration de Roc de Combe dans le groupe Moustérien de débitage Discoïde stricto sensu défini par V. Mourre (Mourre 2003). La prédominance des denticulés et le très faible taux de racloirs $(<5 \%)$ inscrivent par ailleurs cette série dans l'ensemble 1 défini par $\mathrm{C}$. Thiébaut regroupant des niveaux du Moustérien Discoïde à denticulés de divers sites rapportés à l'OIS 3 avec une exploitation monospécifique de la faune (Thiébaut 2005).

\section{2 - Étude comparative}

Plusieurs points méritent un approfondissement afin d'intégrer le Moustérien de Roc de Combe dans son contexte régional d'une part, dans les problématiques 


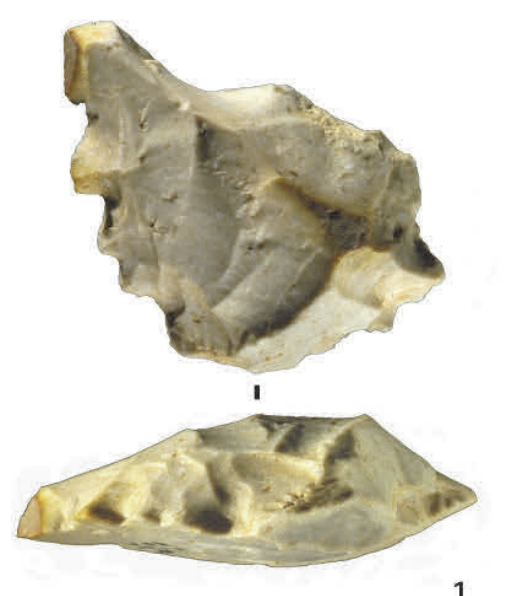

1

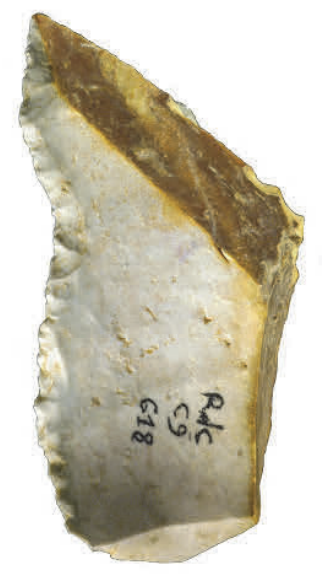

3

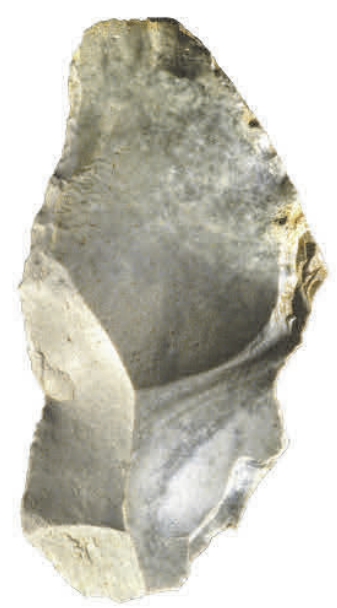

6
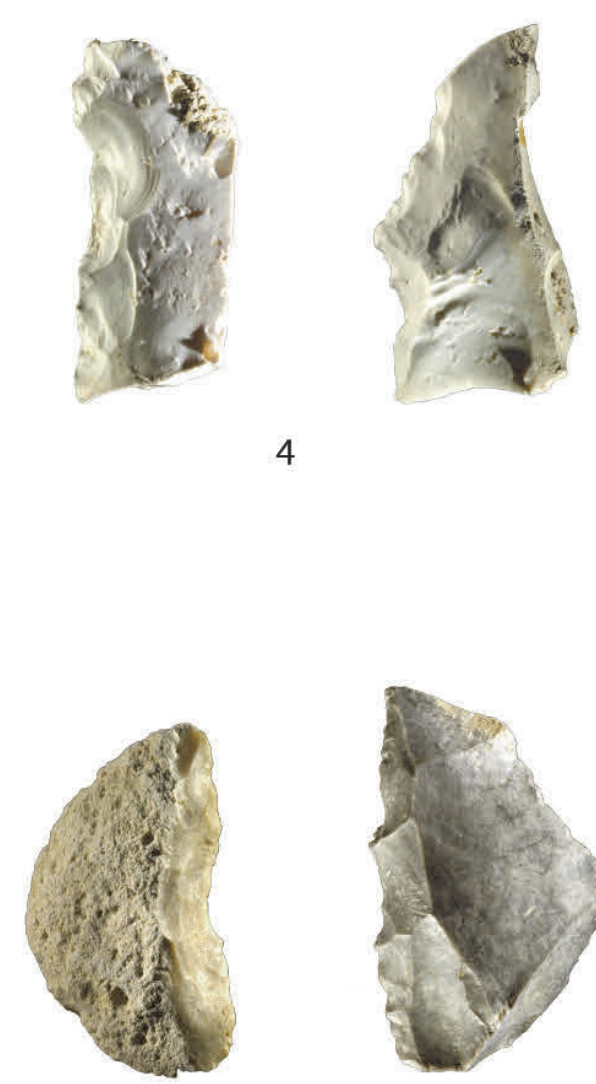

4

5

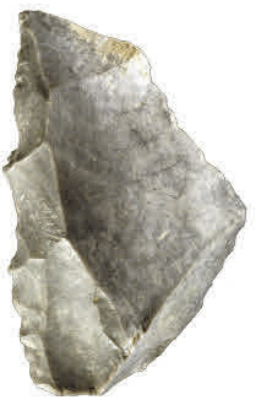

8
Figure 10 - 1 à 5 : Roc de Combe (Lot), Moustérien Discoïde à denticulés. Outillage retouché en calcédoine tertiaire. 1 et 2 : pointes pseudo-Levallois à denticulations moyennes. 3 : éclat à dos cortical opposé à une micro-denticulation. 4 : éclat débordant à moyennes et macro-denticulations opposées au dos. 5 : éclat débordant à microdenticulations opposées au dos. 6 à 8 : outillage retouché en silex sénonien : 6 : éclat cortical présentant une retouche composite (racloir et denticulé). 7 : macroencoche clactonienne sur un éclat (> 50\% cortex) à dos naturel. 8 : éclat débordant à moyennes et microdenticulations opposées au dos. (c) MNP Les Eyzies - Dist. RMNcliché Ph. Jugie.

Figure 10 - Roc de Combe (Lot), Moustérien Discoïde à denticulés. 1 to 5: tool group in Cenozoic calcedony. 1 and 2: Middle size denticulation denticulate pseudoLevallois points. 3: microdenticulate cortical backed flake. 5 : microdenticulate débordant flake. 6 to 8: Senonian flint tool group. 6: mixed tool (scraper and denticulate). 7: clactonian macro-notched cortical backed flake. 8: Middle size denticulation and microdenticulation denticulate debordant flake. Credits: MNP Les Eyzies - Dist. RMN-photo Ph. Jugie. actuelles traitant des techno-complexes de la fin du Paléolithique moyen (LMP) d'autre part.

La diversité morphotechnique des supports obtenus en fin de séquence, qu'elle soit au niveau des nucléus ou des produits, traduit une certaine variabilité du débitage Discoïde. Cette dernière a fait l'objet de recherches récentes qui montrent que le concept Discoïde régit plusieurs modalités de débitage et des structurations différentes du schéma opératoire (Mourre 2003 ; Slimak 2003 ; Bourguignon, Faivre, Turq 2004 ; Locht 2004 ; Faivre 2008). Les séquences de débitage sont courtes ou longues et ont eu recours à des modalités différentes, qui ne sont pas uniquement centripètes (uni ou bifaciale) selon la 


\begin{tabular}{|c|c|c|c|c|c|c|c|c|c|c|}
\hline \multirow{2}{*}{ Outils } & \multicolumn{2}{|c|}{ Calcédoine tertiaire } & \multicolumn{2}{|c|}{ Silex sénonien } & \multicolumn{2}{|c|}{ Quartzites } & \multicolumn{2}{|c|}{ Autres MPL } & \multirow{2}{*}{$\begin{array}{l}\text { Total par } \\
\text { type de } \\
\text { retouche }\end{array}$} & \multirow[t]{2}{*}{$\%$} \\
\hline & Effectif & $\%$ & Effectif & $\%$ & Effectif & $\%$ & Effectif & $\%$ & & \\
\hline DENTICULÉS & 175 & $47,4 \%$ & 72 & $57,6 \%$ & 6 & $60,0 \%$ & 4 & $40,0 \%$ & 257 & $50,0 \%$ \\
\hline ENCOCHES & 79 & $21,4 \%$ & 18 & $14,4 \%$ & 3 & $30,0 \%$ & 2 & $20,0 \%$ & 102 & $19,8 \%$ \\
\hline DENT repris & 2 & $0,5 \%$ & & & & & & & 2 & $0,4 \%$ \\
\hline ENC repris & 1 & $0,3 \%$ & & & & & & & 1 & $0,2 \%$ \\
\hline RACLOIRS & 17 & $4,6 \%$ & 6 & $4,8 \%$ & & & 1 & $10,0 \%$ & 24 & $4,7 \%$ \\
\hline MIXTES & 29 & $7,9 \%$ & 10 & $8,0 \%$ & & & 3 & $30,0 \%$ & 42 & $8,2 \%$ \\
\hline $\begin{array}{l}\text { FRAGMENT } \\
\text { D'OUTIL }\end{array}$ & 13 & $3,5 \%$ & 2 & $1,6 \%$ & 1 & $10,0 \%$ & & & 16 & $3,1 \%$ \\
\hline $\begin{array}{l}\text { RETOUCHE } \\
\text { PARTIELLE }\end{array}$ & 12 & $3,3 \%$ & 5 & $4,0 \%$ & & & & & 17 & $3,3 \%$ \\
\hline NON TYPABLE & 10 & $2,7 \%$ & 1 & $0,8 \%$ & & & & & 11 & $2,1 \%$ \\
\hline $\begin{array}{l}\text { QUELQUES } \\
\text { RETOUCHES }\end{array}$ & 29 & $7,9 \%$ & 4 & $3,2 \%$ & & & & & 33 & $6,4 \%$ \\
\hline RETOUCHE ? & 2 & $0,5 \%$ & 7 & $5,6 \%$ & & & & & 9 & $1,8 \%$ \\
\hline TOTAL & 369 & $100 \%$ & 125 & $100 \%$ & 10 & $100 \%$ & 10 & $100 \%$ & 514 & $100 \%$ \\
\hline
\end{tabular}

Tableau 4 - Roc de Combe (Lot). Effectifs et proportion des différents types de retouche par matière première.

Table 4 - Roc de Combe (Lot). Number and proportion of different retouch types by raw material.
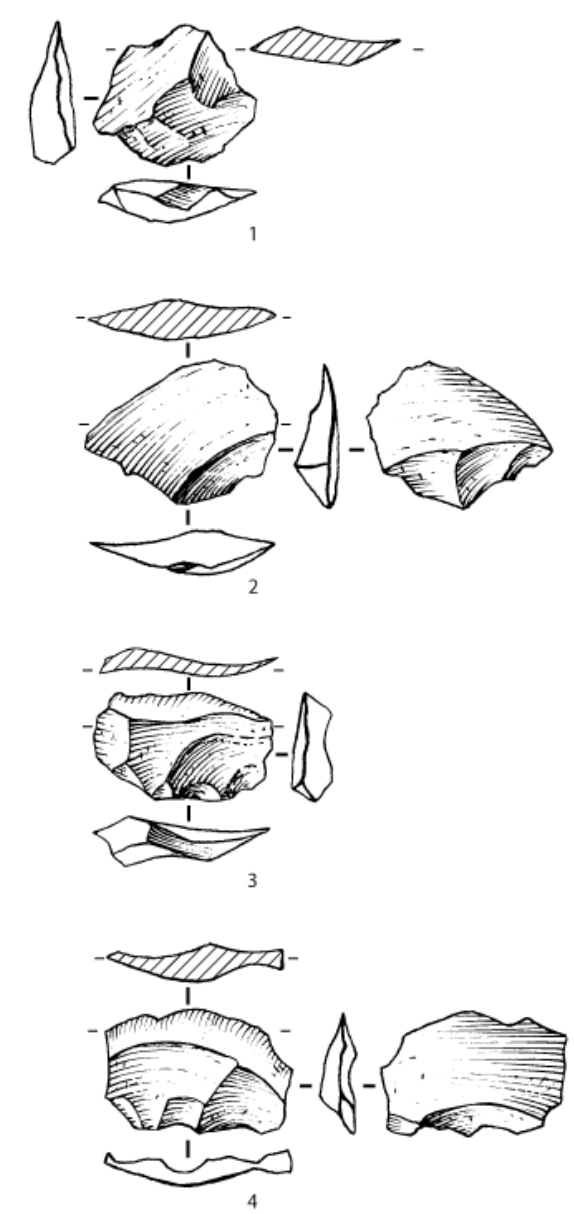

définition classique (Boëda, Geneste, Meignen 1990 ; Boëda 1993 ; Meignen 1993). La présence de différents types de supports mis en œuvre en liaison avec les matières premières exploitées pose la question de la structuration de la chaîne opératoire. Plusieurs structurations ont été documentées pour le Paléolithique moyen, dont les trois principales (résumées in Delagnes 2010) sont la ramification, le recyclage et la « structure scalariforme » (Geneste 1991 ; Bourguignon, Faivre, Turq 2004). L'évaluation des finalités de débitage et leurs caractères morpho-techniques, ainsi que les modalités engagées pour chaque chaîne opératoire sont importantes à discerner puisqu'elles impliquent une économie de débitage et un degré d'anticipation différents. À Roc de Combe, la méthode Discoïde est exclusive quels que soient les différents matériaux mis en œuvre, silex et quartzites, avec uniformisation des méthodes, modalités et techniques rappelant en cela le cas de Mauran (Jaubert 1994). La notion d'économie différentielle de débitage - qui implique une gestion tout aussi différentielle en fonction de la matière première (Perlès 1991) - ne peut être évoquée ici.

Figure 11 - Roc de Combe (Lot), Moustérien Discoïde à denticulés. Différents types d'éclats d'encoche. 1 : éclat d'encoche non cortical. 2 : éclat d'encoche extrait à partir de la face inférieure. 3 : éclat de reprise d'encoche non cortical. 4 : éclat de reprise de denticulé. Dessins : G. Devilder.

Figure 11 - Roc de Combe (Lot), Denticulate Discoïd Mousterian. Different types of notch's flakes. 1: non cortical notch's flake. 2: notch's flake from the inferior surface flake. 3: re-sharpening flake of non cortical notch. 4: re-sharpening flake of denticulate. Drawings: G. Devilder. 
Cependant, notons que des différences existent par exemple dans la longueur des séquences et la finalité du débitage : les nucléus en silex sénonien témoignent par exemple d'une gestion optimale, plus soignée dans l'aménagement de la charnière et des convexités, dans la mesure où c'est principalement la réduction des modules qui a provoqué l'arrêt du débitage. Les supports favorisés pour l'exploitation sont des éclats corticaux. L'hypothèse de leur importation en vue de leur débitage peut être suggérée. L'analyse des produits ne montre pas une différenciation morphotechnique forte qui serait fonction des types de nucléus : les finalités de débitage sont identiques pour les différents types de nucléus et de matériaux. Des blocs et des éclats tirés de ces blocs sont exploités selon la méthode Discoïde, afin de produire des éclats corticaux (utilisés comme outils ou comme nucléus) ainsi que des éclats prédéterminés et prédéterminants. II en découle une décroissance dimensionnelle au fur et à mesure de la production, pour des supports similaires. Le principe mis en œuvre est celui de la récurrence sur différentes générations de produits (Geneste 1991), ou « récurrence "diminutive" et homothétique de la production » (Delagnes 2010). Ce principe est employé jusqu'au maximum de ses possibilités comme en témoignent des nucléus aménagés en denticulés $(n=5)$. De même, des outils encochés, par la taille des encoches, posent la question d'un micro-débitage, documenté par exemple à Champs de Bossuet et à CombeGrenal (Bourguignon et Turq 2003 ; Bourguignon, Faivre, Turq 2004).

\section{3 - Datations}

Plusieurs dates radiocarbone ont été obtenues dans les années 1990 concernant la séquence du Paléolithique supérieur de Roc de Combe (Hedges et al. 1990). Une bonne partie des incohérences apparues alors sont expliquées par les mélanges mis en évidence lors de l'analyse taphonomique des industries lithiques (Bordes 2002, p. 95). D'autres incohérences sont liées, elles, à la modification des labels des couches entre la fouille et la publication (ibidem). Dans ce contexte, de nouvelles dates furent tentées sur du matériel issu de carrés et de couches où avaient été reconnue l'interstratification. Ce travail fut réalisé en 2002 par $\mathrm{H}$. Valladas, dans le cadre du projet Eclipse (coord. M.-F. Sánchez Goñi). Les pièces datées sont des os non brûlés, repérés par carrés et par couche. Elles ont permis de confirmer l'existence de mélanges dans cette zone du gisement (tabl. 5).

Compte tenu du contexte archéologique dans lequel elles ont été réalisées, il est très probable que certains de ces résultats sont à rapporter aux occupations moustériennes (tabl. 5). Les plus anciennes de ces dates étant de l'ordre de 45-50 ka BP, il se confirme que ce Moustérien Discoïde à denticulés de Roc de Combe est d'âge très récent. Ce résultat est en bonne cohérence avec les caractéristiques techno-typologiques de la série et les âges obtenus pour d'autres techno-complexes analogues du Sud-Ouest de la France (synthétisés in Guibert et al. 2008).

\section{4 - Bilan techno-économique : le Moustérien de Roc-de-Combe dans son contexte régional}

L'acquisition est très majoritairement locale avec 97,8 \% des matériaux provenant d'une zone inférieure à $10 \mathrm{~km}$ de distance : calcédoine tertiaire, silex du Sénonien, quartzites, etc. Une infime partie des matières lithiques (0,05\%) provient de plus de $40 \mathrm{~km}$ de Roc de Combe, jusque $70 \mathrm{~km}$ (silex du Bergeracois et du Fumélois). La comparaison avec des gisements quercynois voisins, Les Fieux, couches G7 et K et La Borde et du Périgord, CombeGrenal couches 12 et 20 , où une industrie moustérienne à denticulés a été identifiée (Bordes 1972 ; Jaubert et al. 1990 ; Faivre 2002, 2006, 2008 ; Thiébaut 2005 ; Faivre et al. 2013) montre que la présence de matières allochtones comme le Fumélois n'y a jamais été signalée. En effet, le site des Fieux atteste un approvisionnement local ou régional $(<12 \mathrm{~km}$ pour la couche $\mathrm{K}$ et $<25-30 \mathrm{~km}$ pour la couche G7 qui comprend ces silex du haut-Agenais : Turq 1992, 2000) par l'éloignement des sources de matières premières ainsi qu'un circuit inhabituel. Ceci dit, les sites publiés étant essentiellement quercynois (Chalard et al. 2007), Roc-de-Combe n'occuperait-il pas un statut particulier en pays bourian, entre Dordogne et Quercy ?

\begin{tabular}{|c|c|c|c|c|}
\hline $\begin{array}{l}\text { Nom du } \\
\text { carré / déc }\end{array}$ & $\begin{array}{l}\text { Couche } \\
\text { d'après } \\
\text { sachets }\end{array}$ & $\begin{array}{c}\text { Nom de la } \\
\text { date }\end{array}$ & Âge BP & Contenu après tapho \\
\hline $\mathrm{H} 10$ & 10 & OxA 1264 & $31000 \pm 750$ & \multirow{2}{*}{$\begin{array}{l}\text { Moustérien + qq } \\
\text { éléments du PS }\end{array}$} \\
\hline $\mathrm{H} 10$ & 10 & OxA 1443 & $38000 \pm 2000$ & \\
\hline G8 & 1a & OxA 1254 & $32000 \pm 1000$ & $\begin{array}{l}\text { Mélange généralisé, du } \\
\text { Gravettien au } \\
\text { Moustérien }\end{array}$ \\
\hline G10 & B.1 & GifA 101261 & $50000 \pm 2400$ & \multirow{3}{*}{$\begin{array}{l}\text { Mélange généralisé de } \\
\text { Aurignacien, } \\
\text { Châtelperronien, } \\
\text { Moustérien }\end{array}$} \\
\hline G10 & B.1 & GifA 101262 & $44700 \pm 2900$ & \\
\hline F8 & $\mathrm{C}$ & GifA 101263 & $35210 \pm 550 . \mathrm{BP}$ & \\
\hline K9 & 8 & GifA 101264 & $39540 \pm 970$ & \multirow{3}{*}{$\begin{array}{l}\text { Châtelperronien avec } \\
\text { Moustérien Levallois à } \\
\text { la base }\end{array}$} \\
\hline K9 & 8 & GifA 101265 & $45100 \pm 2100$ & \\
\hline K9 & 8 & GifA 101266 & $40000 \pm 1300$ & \\
\hline
\end{tabular}

Tableau 5 - Roc de Combe (Lot). Datations ${ }^{14} \mathrm{C}$. Travail réalisé par H. Valladas, en collaboration avec F. d'Errico, dans le cadre du projet Eclipse du programme Excursion (coord. M.-F. Sánchez-Goñi). Les pièces datées sont des os non brûlés, repérées par carré et par couche.

Table 5 - Roc de Combe (Lot). ${ }^{14} \mathrm{C}$ data. Realized by H. Valladas, in collaboration with F. d'Errico, in the Eclipse project of the Excursion program (coord. M.-F. Sánchez-Goñi). The dated objects are unburned bones, marked with square and layer. 
Ceux de Combe-Grenal, qui est également local $(<12 \mathrm{~km})$ ou encore celui de La Borde, chronologiquement plus ancien et géographiquement plus éloigné, comporte un territoire d'approvisionnement étendu à une quarantaine de kilomètres. Plusieurs auteurs proposent une circulation de matériel (les Moustériens emportant avec eux un stock de matière première, consommée et abandonnée durant leurs déplacements) (Turq 1992, 2000 ; Faivre et al. 2013) d'ouest en est, du Périgord vers le Quercy et vice versa dans de très faibles proportions. Elle pourrait alors coïncider avec les déplacements saisonniers des grands mammifères (Chalard et al. 2007 ; Turq et Morala op. cit.). Aucun approvisionnement en provenance du haut-Agenais vers les causses quercynois n'a été jusqu'à présent attesté. Excepté probablement pour Combe-Grenal, Roc-deCombe se démarquerait donc des autres sites régionaux.

L'exploitation des nucléus ne requiert pas une phase de préparation élaborée (ou décorticage) puisque le débitage Discoïde permet d'obtenir très rapidement des convexités et des surfaces de débitage idéales pour la production de produits de différents types, d'où un chevauchement probable entre les phases d'initialisation stricte et de production. Ces deux phases sont également confondues pour les galets de quartzite : les propriétés physiques du néocortex fournissent des plans de frappe optimaux, comme démontré depuis longtemps (Tavoso 1978). Les convexités naturelles qu'offre la matière première locale ou obtenues rapidement entraînent la concomitance de ces deux phases qui servent au moins deux objectifs : production de grands éclats corticaux destinés à l'outillage (brut ou retouché) et production d'éclats similaires alloués à une seconde phase de production.

Le déficit de certaines catégories de supports (éclats de type Kombewa et pointes pseudo-Levallois au sein des matières dominantes) témoignerait d'une stratégie d'exportation préférentielle. Ils constitueraient alors des produits d'intention première destinés à l'exportation. Les caractéristiques de l'industrie en silex minoritaire montrent que diverses matières ont transité par Roc-de-Combe. Rares et éloignées, elles constituent un marqueur aisément identifiable pour attester des déplacements. Ce n'est pas le cas des industries en roches dominantes qui peuvent avoir fait l'objet de transports courts (ou plus longs) sans laisser d'indices d'un tel traitement.

Un comportement de recyclage peut être également envisagé par la présence d'une série de pièces à double patine $(n=27)$ : elles ont été abandonnées puis réutilisées bien après leur premier dépôt.

Les chiffres bruts nous montrent que toutes les séquences de la chaîne opératoire (Geneste 1985) sont documentées in situ, depuis le décorticage jusque l'abandon. Toutefois, il convient d'être prudent car ils pourraient dissimuler un comportement plus complexe : celui de l'importation de supports débités ailleurs et qui ont fait l'objet d'une circulation et d'une exploitation dynamiques sans respect de l'ordonnance plus rigide de la chaîne opératoire classique. La diversité de caractères physiques des pièces elles-mêmes, au-delà des décomptes classiques, devient alors un critère essentiel dans l'optique d'une reconstitution de la chaîne opératoire.

\section{Conclusion et perspectives}

Le contexte géologique local de la Bouriane, moins pourvu que son voisin périgourdin (Sarladais, Périgord noir) en matières premières de haute qualité n'a pas empêché les Moustériens de s'en satisfaire pour leur approvisionnement principal. Ils ont opté pour un concept de débitage reproductible sur tous types de matériaux (silex sénonien ou tertiaire, quartz/métaquartzites). Cela dénote une certaine capacité d'adaptation, une grande mobilité, éventuellement à l'intérieur d'un territoire assez restreint (non perceptible à travers les seules matières premières lithiques) dont il est toujours difficile d'en estimer les modalités (Faivre 2002 ; Delagnes 2010 ; Delagnes et Rendu 2011). De ce fait, les séquences de débitage sont indifféremment courtes ou longues, l'approvisionnement en matières premières ne constituant apparemment pas une contrainte. La production est orientée vers l'obtention de supports de morphologie plus ou moins standardisée, à savoir des éclats asymétriques de section et de profil comportant un dos opposé à un tranchant, ainsi que des éclats corticaux, ne nécessitant pas d'excellentes matières premières en terme d'aptitude à la taille. Quelques pièces d'origine plus lointaine témoignent de déplacements ou d'échanges occasionnels d'objets jusqu'à une distance d'environ $70 \mathrm{~km}$.

Cette série de Roc de Combe rejoint la liste des sites paléolithiques moyen de débitage Discoïde et outils denticulés qui se placent en position quasi terminale du Paléolithique moyen récent dans le Sud-Ouest de la France (Thiébaut 2005 ; Jaubert et al. 2011 ; Jaubert 2012), tous plus récents que le MTA tel qu'il est classiquement défini régionalement (Soressi 2005) quand ce dernier est attesté dans les séquences (SaintCésaire, Le Moustier, La Quina), ce qui n'est apparemment pas le cas à Roc de Combe, sinon à l'état d'indices non validés. Si l'on s'en tient aux séries publiées de ce Moustérien Discoïde à denticulés, parfois datées comme Saint-Césaire Egf/c.11-12 (42,4 \pm 4,3 en TL : Mercier et al. 1993) La Quina c.6a (43,3 $\pm 3,6$ en TL : Debénath et Jelinek 1999) et les Rochers de Villeneuve c.N (> 45,2 $\pm 1,1$ AMS : Beauval et al. 2006), ou non datées (Hauteroche, Sandougne, Brouillaud CombeGrenal, Chadourne, Les Fieux) et sans prendre en compte les niveaux du Moustier potentiellement Discoïde à denticulés (c. I-H) également contemporains et en cours de révision (Gravina et Discamps 2013), ce technocomplexe, même s'il succède au MTA, relève de la même biozone du stade isotopique 3 (Discamps, Jaubert, Bachellerie 2011) (fig. 12).

Ce Moustérien Discoïde à denticulés est clairement antérieur à l'événement Heinrich 4 (38,6-40,2 calBP) et très vraisemblablement contemporain des interstades Gl 9-11 (40,2-43,3 calBP). L'étude de la faune associée est 


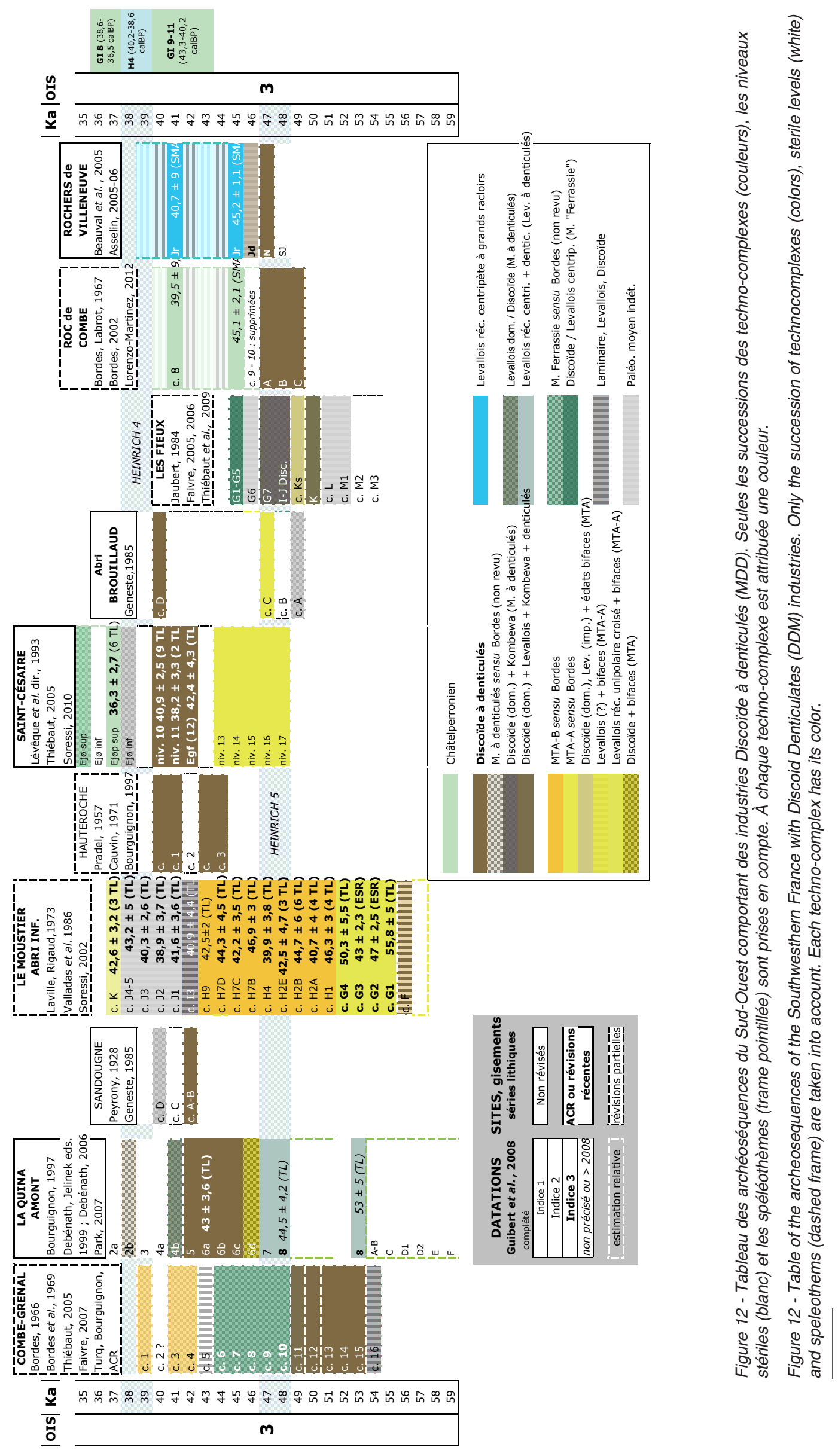


désormais primordiale pour confirmer cette attribution chrono-climatique (Discamps et al. 2013).

Enfin, le dernier point de discussion est la présence ou non, à Roc de Combe de quelques éclats Levallois rentrant dans la variabilité d'une ultime occurrence Paléolithique moyen final, cette fois postérieure au Moustérien Discoïde à denticulés telle que signalée aux Rochers de Villeneuve (Asselin 2005 ; Jaubert et al. 2011), vraisemblablement dans d'autres séquences de Charente (Les Plumettes : Airvaux, Beauval, Primault 2012 ; Discamps, Jaubert, Bachellerie 2011) et, sous réserve des réévaluations en cours dans d'autres séquences aquitaines. À Roc de Combe, ce Moustérien Discoïde à denticulés se place en effet stratigraphiquement en dessous d'une discrète série d'éclats Levallois (Pelegrin 1995), eux-mêmes surmontés par du Châtelperronien (Bordes 2002). La réévaluation systématique des séquences du Sud-Ouest de la France conduit désormais à rejeter le modèle affiliant le MTA-A, puis B et le Châtelperronien (Soressi 2002) remplacé par un autre modèle (Gravina et Discamps 2013). Des recherches récentes avancent au contraire l'hypothèse d'une rupture nette et dépourvue de stade transitionnel entre le fonds Paléolithique moyen récent $(\mathrm{MTA}-\mathrm{A} / \mathrm{B} \rightarrow \mathrm{M}$. Discoïde à denticulés $\rightarrow M$. Levallois à grands racloirs) et le début du Paléolithique supérieur (Bachellerie 2011 ; Bordes et al. 2011 ; Jaubert et al. 2011). Ce sont donc des technocomplexes sans liens techniques évidents qui se succèdent depuis la fin du Paléolithique moyen, jusqu'au début du Paléolithique récent remettant sérieusement en cause toute hypothèse sur l'existence même d'industries dites « de Transition ». Le Moustérien de Roc de Combe contribue incontestablement à étayer ce nouveau schéma archéostratigraphique. II s'agit dorénavant de mieux caractériser la variabilité et la chronologie des ultimes cultures moustériennes, qui ont immédiatement précédé voire accompagné (?) les débuts du Châtelperronien.

\section{Remerciements}

Nos remerciements vont à Jean-Jacques Cleyet-Merle, directeur du MNP et à son personnel pour nous avoir autorisé (M.L.M.) à étudier cette série dans le cadre d'un mémoire de master 2 de l'université Bordeaux 1. En plus de leurs missions muséales dans le même établissement, un merci tout particulier à André Morala et Alain Turq pour leurs conseils scientifiques toujours précis et avisés, notamment pour la détermination des matières premières. Et, d'une manière générale au personnel du musée pour les conditions d'accueil et l'aide substantielle dans le conditionnement, le lavage - marquage d'une partie de cette série (Bernard Nicolas et Peggy Jacquement). Un merci très sincère des trois auteurs à Jean-Philippe Rigaud pour son accueil toujours bienveillant et désintéressé pour nos travaux, et ici ses informations relatives à ses souvenirs de fouille à Roc de Combe. Remercions également Gauthier Devilder (CNRS MOM-Lyon et PACEA) pour ses excellents dessins au trait. Enfin, merci aux trois rapporteurs pour leurs conseils avisés, appropriés et complémentaires qui ont contribué à améliorer la version initiale du manuscrit.

\section{Références bibliographiques}

AIRVAUX J., BEAUVAL C. et PRIMAULT J. 2012 - Le site du Moustérien récent de La Ganne à Mazerolles et les repaires d'hyènes des Plumettes et des Rochers de Villeneuve à Lussac-les-Châteaux (Vienne) : hypothèses sur la relation homme-carnivores. Préhistoire du SudOuest, vol. 20, $\mathrm{n}^{\circ} 1$, p. 3-37.

ASSELIN G. 2005 - Étude techno-typologique et spatiale du matériel lithique du niveau $J$ du site Moustérien des Rochers de Villeneuve, Lussac-les-Châteaux, Vienne (86). Mémoire de master 2, Université Bordeaux 1, $75 \mathrm{p}$.

ASTRUC J.-G. 1990 - Carte géologique de la France. Gourdon $n^{\circ}$ 832. Carte $1 / 50000^{\circ}$. Orléans : éditions du BRGM.

BACHELLERIE F. 2011 - Quelle unité pour le Châtelperronien ? Apport de l'analyse taphonomique et techno-économiques des industries lithiques de trois gisements aquitains de plein-air : le Basté, Bidart (Pyrénées-Atlantiques) et Canaule II (Dordogne). Thèse de doctorat, Université Bordeaux 1, $442 \mathrm{p}$.

BEAUVAL C., LACRAMPE-CUYAUBÈRE F., MAUREILLE B. et TRINKAUS E. 2006 - Direct radiocarbon dating and stable isotopes of the neandertal femur from Les Rochersde-Villeneuve (Lussac-les-Châteaux, Vienne), Bulletins et mémoires de la Société d'Anthropologie de Paris, 18 (1-2).

BOËDA É. 1993 - Le débitage discoïde et le débitage Levallois centripète récurrent. Bulletin de la Société Préhistorique Française, 90, 6, p. 392-404.

BOËDA É. 1995 - Caractéristiques techniques des chaînes opératoires des niveaux micoquiens de Külna (Tchécoslovaquie). In : Les industries à pointes foliacées d'Europe centrale. Actes du colloque de Miskolc (10-15 septembre 1991). Paleo, supplément $n^{\circ} 1$, p. 57-72.

BOËDA É., GENESTE J.-M. et MEIGNEN L. 1990 Identification de chaînes opératoires lithiques du Paléolithique ancien et moyen. Paleo, 2, p. 43-80.

BORDES F. 1961 - Typologie du Paléolithique ancien et moyen. Bordeaux : Imprimerie Delmas, Publ. de l'Institut de Préhistoire de l'Université de Bordeaux, 2 t., 85 p. 108 pl. h.t.

BORDES F. 1968 - La question périgordienne. In : la Préhistoire, problèmes et tendances. Paris : éditions du CNRS, p. 59-70.

BORDES F. 1972 - A tale of two caves. New York: Harper \& Row. p. 169.

BORDES F. 1981 - Vingt cinq ans après : le complexe moustérien revisité. Bulletin de la Société Préhistorique Française, 78, 3, p. 103-108.

BORDES F. 1984 - Leçons sur le Paléolithique. Tome 2 : le Paléolithique en Europe. Paris : éditions du CNRS (Cahiers du Quaternaire, 7). 
BORDES F. et LABROT J. 1967 - La stratigraphie du gisement du Roc-de-Combe (Lot) et ses implications. Bulletin de la Société Préhistorique Française, LXIV, 1, p. 15-28.

BORDES J.-G. 2002 - Les interstratifications Châtelperronien/Aurignacien du Roc-de-Combe et du Piage (Lot, France). Analyse taphonomique des industries lithiques ; implications archéologiques. Thèse de doctorat, Université Bordeaux 1, $421 \mathrm{p}$.

BORDES J.-G., BACHELLERIE F., LE BRUN-RICALENS F. et MICHEL A. 2011 - Towards a new 'transition': New data concerning the lithic industries from the beginning of the Upper Palaeolithic in Southwestern France. In : A.P. Derevianko \& M. Shunkov Eds., Characteristc features of the Middle to Upper Palaeolithic Transition in Eurasia, Proceeding of the International Symposium, "Characteristc features of the Middle to Upper Palaeolithic Transition in Eurasia : Development of Culture and Evolution of Homo Genus“ (July 4-10, Denisova Cave, Altai), Novosibirsk, Department of the Institute of Archaeology and Ethnography SB RAS, p. 116-129.

BOURGUIGNON L. 1997 - Le Moustérien de type Quina : nouvelle définition d'une technique. Thèse de doctorat, Université de Paris X-Nanterre, 2 t., 672 p.

BOURGUIGON L. et TURQ A. 2003 - Une chaîne opératoire de débitage Discoïde sur éclat du Moustérien à denticulés aquitain : les exemples de Champ Bossuet et de Combe-Grenal c. 14 ; In: M. Peresani (Ed.), Discoid lithic technology. Advances and implications. BAR International series, 1120, p. 131-152.

BOURGUIGNON L., FAIVRE J.-Ph et TURQ A. 2004.Ramification des chaînes opératoires : une spécificité du Moustérien ? Paleo, n 16, p. 37-48.

BRENET M. et FOLGADO M. 2003 - Le débitage Discoïde du gisement des Forêts à Saint-Martin-de-Gurçon (Dordogne). In: M. Peresani (Ed.), Discoid Lithic technology. Advances and implications. BAR International Series, 1120, p. 153-177.

CHALARD P., FAIVRE J.-Ph., JARRY M., JAUBERT J., MOURRE V. et TURQ A. 2007 - Espaces du Paléolithique moyen : témoins d'utilisation de silex allochtones en Quercy (France). In : J. Évin (Dir.), Un siècle de construction du discours scientifique en Préhistoire, volume I "Des idées $d^{\prime}$ hier... ». 26 congrès préhistorique de France. Congrès du Centenaire, Avignon-Bonnieux (21-25 septembre 2004). Paris : SPF, p. 217-235.

CHAMPAGNE et F. ESPITALIÉ R. 1967 - La stratigraphie du Piage : note préliminaire. Bulletin de la Société Préhistorique Française. 64, p. 29-34

DEBÉNATH A. et JELINEK A. J. (Dir.) 1999 - Nouvelles fouilles à La Quina (Charente). Résultats préliminaires. Gallia Préhistoire, T. 40, p. 29-74.
DELAGNES A. 2010 - Systèmes techniques et mobilité au Paléolithique moyen : interaction et implications diachroniques. Thèse d'Habilitation à Diriger des Recherches : Université Bordeaux 1, 229 p.

DELAGNES A. et RENDU W. 2011 - Shifts in Neandertal mobility, technology and subsistence strategies in Western France. Journal of Archaeological Science, 38, p. 1771-1783.

DISCAMPS E., JAUBERT J. et BACHELLERIE F. 2011 Human choices and environmental contraints : deciphering the variability of large game procurement from Mousterian to Aurignacian times (MIS 5-3) in southwestern France. Quaternary Science Reviews, 30, 19-20, p. 2755-2775.

DISCAMPS E., SOULIER M.-C., BACHELLERIE F., BORDES J.-G., CASTEL J.-Chr. et MORIN E. 2013 - Des faunes et des hommes : interactions entre environnements et cultures dans le Sud-Ouest de la France. In : J. Jaubert, $\mathrm{N}$. Fourment et P. Depaepe, Transitions, ruptures et continuité en Préhistoire, XXVIle congrès préhistorique de France - Bordeaux-Les Eyzies, 31 mai-5 juin 2010, p. 299-314.

FAIVRE J.-Ph. 2002 - Approche technologique du Moustérien du site des Fieux (Miers, Lot). Études des industries lithiques du niveau G7 (locus 1). Mémoire de maîtrise de l'Université de Toulouse-Le Mirail, 126 p.

FAIVRE J.-Ph. 2004 - L'industrie lithique moustérienne du niveau G7 des Fieux (Miers, Lot) : mobilité humaine et diversité des compétences techniques. Paleo, t. 16, p. 71-90.

FAIVRE J.-Ph. 2006 - L'industrie moustérienne du niveau Ks (locus 1) des Fieux (Miers, Lot) : mobilité humaine et diversité des compétences techniques. Bulletin de la Société Préhistorique Française, 103, 1, p. 17-32.

FAIVRE J.-Ph. 2008 - Organisation techno-économique des systèmes de production dans le Paléolithique moyen récent du Nord-est Aquitain : Combe Grenal et Les Fieux. Thèse de doctorat, Université Bordeaux 1, 2008, 555 p.

FAIVRE J.-Ph., TURQ A., BOURGUIGNON L., BISMUTH T., COLONGE D., DEMARS P.-Y., JARRY M. et JAUBERT J., 2013 - Le Paléolithique moyen du Quercy : comportements techno-économiques et variabilités des productions lithiques. In : M. Jarry, J.-Ph. Brugal et C. Ferrier (Dir.), Modalités d'occupations et exploitation des milieux au Paléolithique dans le Sud-Ouest de la France : l'exemple du Quercy, Actes session C67, XV'̀̀me Congrès de I'UISPP, Lisbonne, sept. 2006, Suppl. à Paleo, $\mathrm{n}^{\circ} 4$, p. $197-235$.

GENESTE J.-M. 1985 - Analyse lithique d'industries moustériennes du Périgord: une approche technologique du comportement des groupes humains au Paléolithique moyen. Thèse de doctorat, Université de Bordeaux I, 2 t., $572 \mathrm{p}$. 
GENESTE J.-M. 1988 - Les industries de la grotte Vaufrey : technologie du débitage, économie et circulation de la matière première lithique, In : J.-Ph. Rigaud dir., La grotte Vaufrey. Paléoenvironnement. Chronologie. Activités humaines, Mémoire de la Soc. Préh. Franç., XIX, Paris, p. 441-517.

GENESTE J.-M. 1991 - Systèmes techniques de production lithique : variation techno-économique dans les processus de réalisation des outillages paléolithiques. Techniques et cultures. "Préhistoire et technologie. Le geste retrouvé », 17-18, p. 1-35.

GRAVINA B. et DISCAMPS E. 2013 - MTA B or not to 'B', that is the question? Poster ESHE September 2013, Wien.

GRAVINA B., BACHELLERIE F., DISCAMPS E., BORDES J.-G. et JAUBERT J. 2012 - A new ending for the Mousterian in south-western France ? A revision of the Final Middle Paleolithic record in South-western France and its implications. Proceeding of the European Society for the study of Human Evolution, $2^{\text {nd }}$ annual meeting, Bordeaux, 21-22 Sept. 2012, p. 89.

GRAYSON D.K. et DELPECH F. 2008 - The large mammals of Roc de Combe (Lot, France): the Chatelperronian and Aurignacian assemblages. Journal of Anthropological Archaeology, 27, p. 338-362.

GUIBERT P., BECHTEL F., BOURGUIGNON L., BRENET M., COUCHOUD I., DELAGNES A., DELPECH F., DETRAIN L., DUTTINE M., FOLGADO M., JAUBERT J., LAHAYE Ch., LENOIR M., MAUREILLE B., TEXIER J.-P., TURQ A., VIEILLVIGNE E. et VILLENEUVE G., 2008 Une base de données pour la chronologie du paléolithique moyen dans le Sud-Ouest de la France. In : J. Jaubert, J.G. Bordes et I. Ortega, Dir., Les sociétés du Paléolithique dans un Grand Sud-Ouest : nouveaux gisements, nouveaux résultats, nouvelles méthodes. Mémoire de la Société préhistorique française, XLVII [Journées SPF, Université Bordeaux 1, Talence, 24-25 novembre 2006], p. 19-40.

HEDGES R. E. M., HOUSLEY R. A., LAW I. A. et BRONK C. R. 1990 - Radiocarbon dates from the Oxford AMS system: Archaeometry datelist 10. Archaeometry, 32, p. 101-108.

INIZAN M.-L., REDURON-BOLLINGER M. et ROCHE H. 1995 - Technologie de la pierre taillée. Meudon : CREP, $199 \mathrm{p}$.

JARRY M., BERTRAN P., COLONGE D., LELOUVIER L.A. et MOURRE V. 2007 - Les Bosses (Lamagdelaine, Lot, France) : un gisement paléolithique moyen antérieur à l'avant-dernier Interglaciaire sur la moyenne terrasse du Lot. Paris, Travaux de la Société Préhistorique Française (7), $158 \mathrm{p}$.
JAUBERT J. 1993 - Le gisement paléolithique moyen de Mauran (Haute-Garonne) : techno-économie des industries lithiques. Bulletin de la Société préhistorique française, 90, p. 328-335.

JAUBERT J. 1994 - Les industries lithiques. In : C. Farizy, F. David et J. Jaubert (Dir.), Hommes et bisons du Paléolithique moyen à Mauran (Haute-Garonne). $X X X^{e}$ supplément à Gallia Préhistoire. Paris : éditions du CNRS, p. 73-167.

JAUBERT J. 2010 - Les archéoséquences du Paléolithique moyen en Poitou-Charentes. In : J. Buisson-Catil et J. Primault (dir.), Préhistoire entre Vienne et Charente Hommes et sociétés du Paléolithique, Association des publications chauvinoises, Chauvigny (Mémoire XXXVIII), p. 51-55.

JAUBERT J. 2012 - Les archéo-séquences du Paléolithique moyen du Sud-Ouest de la France : quel bilan un quart de siècle après François Bordes ? In : F. Delpech et J. Jaubert (Dir.), François Bordes et la Préhistoire. Actes du colloque international François Bordes, Bordeaux, 22-24 avril 2009, éditions du CTHS, coll. Documents Préhistoriques, $n^{\circ} 29$, p. 235-253.

JAUBERT J. et MOURRE V. 1996 - Coudoulous, Le Rescoundudou, Mauran : diversité des matières premières et variabilité des schémas de production d'éclats. In : A. Bietti et S. Grimaldi (Eds.), Reduction Processes (chaînes opératoires) in the European Mousterian. Quaternaria Nova VI, p. 313-41.

JAUBERT J., BORDES J.-G., DISCAMPS E. et GRAVINA B. 2011 - A new look at the end of the Middle Palaeolithic sequence in Southwestern France. In : A.P. Derevianko \& M. Shunkov Eds., Characteristc features of the Middle to Upper Palaeolithic Transition in Eurasia, Proceeding of the International Symposium, "Characteristc features of the Middle to Upper Palaeolithic Transition in Eurasia : Development of Culture and Evolution of Homo Genus“ (July 4-10), Denisova Cave, Altai), Novosibirsk, Department of the Institute of Archaeology and Ethnography SB RAS, p. 102-115.

JAUBERT J., LORBLANCHET M., LAVILLE H., SLOTTMOLLER R., TURQ A. et BRUGAL J.-Ph. 1990 - Les chasseurs d'Aurochs de La Borde. Un site du Paléolithique moyen (Livernon, Lot). Paris : éditions de la Maison des Sciences de l'Homme (dAf 28), 157 p.

KLARIC L. 2003 - L'unité technique des industries à burins du Raysse dans leur contexte diachronique. Réflexions sur la diversité culturelle au Gravettien à partir des données de La Picardie, d'Arcy-sur-Cure, de Brassempouy et du Cirque de la Patrie, Thèse de Doctorat en Préhistoire, Université de Paris I, $427 \mathrm{p}$. 
LAHAYE Ch. 2005 - Nouveaux apports de la thermoluminescence à la chronologie du Paléolithique dans le Sud-Ouest de la France. Essai en milieu hétérogène et en présence de déséquilibres radioactifs dans les séries de l'uranium, Thèse de doctorat de l'Université Michel de Montaigne Bordeaux 3, 428 p.

LAVILLE H. 1969 - Climatologie et chronologie du Paléolithique du Périgord. Étude sédimentologique de dépôts en grottes et sous abris. Marseille, éd. du CNRS, Université de Provence 422 p. (Études quaternaires, 4).

LOCHT J.-L. 2004 - Le gisement paléolithique moyen de Beauvais (Oise). Thèse de doctorat, Université de Lille 1, 2004, 2 t., 209 p + 126 pl.

LOCHT J.-L. et SWINNEN C. 1994 - Le débitage discoïde du gisement de Beauvais (Oise) : aspects de la chaîne opératoire au travers de quelques remontages. Paleo, 6 , p. 89-104.

LORENZO MARTINEZ M. 2012 - Le Moustérien Discoïde à denticulés du Roc-de-Combe (Lot) : caractérisation techno-économique de l'industrie lithique. Mémoire de master 2, Université Bordeaux 1, 84 p.

MEIGNEN L. (Dir.) 1993 - L'abri des Canalettes : un habitat moustérien sur les grands causses (Nant, Aveyron). Fouilles 1980-1986. Paris : éditions du CNRS, 359 p.

MERCIER N., VALLADAS H., JORON J.-L. et REYSS J.-L. 1993 - Datation par thermoluminescence du site préhistorique de la Roche à Pierrot à Saint-Césaire. In : Lévêque F., Backer A.M. \& Guilbaud M. (Eds), Context of a Late Neanderthal. Implications of Multidisciplinary Research for the Transition to Upper Paleolithic Adaptations at Saint-Césaire, Charente-maritime, France, Monographs in World Archaeology $\mathrm{N}^{\circ} 16$, Madison, Prehistory Press, p. 15-23.

MORALA A. 1984 - Périgordien et Aurignacien en HautAgenais : étude d'ensembles lithiques. Toulouse : École des Hautes Études en sciences sociales, Archive d’Écologie préhistorique, 7.

MOURRE V. 1994 - Les industries en quartz au Paléolithique moyen. Approche technologique de séries du Sud-Ouest de la France, Paris-X, Mémoire de Maîtrise, $111 \mathrm{p}$.

MOURRE V. 1996 - Les industries en quartz au Paléolithique. Terminologie, méthodologie et technologie. Paleo, 8, p. 205-223.

MOURRE V. 2003 - Discoïde ou pas Discoïde ? Réflexion sur la pertinence des critères techniques définissant le débitage Discoïde. In : M. Peresani (Ed.), Discoid lithic technology. Advances and implications. BAR International series, 1120 , p. 1-18.
PARK S.J. 2007 - Systèmes de production lithique et circulation des matières premières au Paléolithique moyen récent et final. Une approche techno-économique à partir de l'étude des industries lithiques de La Quina (Charente). Thèse Université Paris X-Nanterre, 335 p.

PELEGRIN J. 1995 - Technologie lithique : le Châtelperronien de Roc-de-Combe (Lot) et de la Côte (Dordogne). Paris : éditions du CNRS, 279 p. (Cahier du Quaternaire, 20).

PERESANI M. (Dir.) 2003 - Discoid Lithic Technology. Advances and Implications. Oxford, British Archaeological Reports, 1120.

PERLÈS C. 1991 - Économie des matières premières et économie du débitage : deux conceptions opposées ? In : 25 ans d'études technologiques en Préhistoire, bilan et perspectives. Juan-les-Pins : éditions APDCA, p. 35-45.

PEYRONY D. 1933 - Les industries «aurignaciennes «dans le bassin de la Vézère. Bulletin de la Société Préhistorique Française 30:543-559.

RICHTER D., DIBBLE H., GOLDBERG P., McPHERRON Sh. J. P., NIVEN L. SANDGATHE D., TALAMO S. et TURQ A. 2012 - The late Middle Palaeolithic in Southwest France: New TL dates for the sequence of Pech de l'Azé IV. Quaternary International, 294, p. 160-167.

RIGAUD J.-Ph. 2000 - Late Neanderthals in the South West of France and the émergence of the Upper Palaeolithic. In : C. B. Stringer, R. N. E. Barton \& J. Cl. Finlayson (Eds.) Neanderthals on the Edge. Papers from a conference marking the 150th anniversary of the Forbes' Quarry discovery, Gibraltar, Gibraltar, 1999, Oxbow Books, p. 27-31.

SÉRONIE-VIVIEN M. et SÉRONIE-VIVIEN M.-R. 1987 Les silex du Mésozoïque nord-aquitain : approche géologique de l'étude des silex pour servir à la recherche. Supplément au Bulletin de la Société Linéenne de Bordeaux, XV.

SLIMAK L. 1999 - La variabilité des débitages discoïdes au Paléolithique moyen, diversité des méthodes et unité d'un concept - L'exemple des gisements de Baume Néron (Soyons, Ardèche) et du Champ-Grand (Saint-Maurice-surLoire, Loire). Préhist. Anthrop. Méditer., 7-8, (1998-1999), p. 75-88.

SLIMAK L. 2003 - Les débitages Discoïdes moustériens : évolution d'un concept technologique. In: M. Peresani (Ed.), Discoid lithic technology. Advances and implications. BAR International series, 1120 , p. 33-65.

SLIMAK L. 2004 - Les dernières expressions du Moustérien entre Loire et Rhône. Thèse Aix-Marseille I, Université de Provence, 649 p. +214 pl. 
SORESSI M. 2002 - Le Moustérien de tradition acheuléenne du Sud-Ouest de la France. Discussion sur la signification du faciès à partir de l'étude comparée de quatre sites : Pech-de-l'Azé I, Le Moustier, La Rochette et la Grotte XVI. Thèse de doctorat, Université Bordeaux 1, $339 \mathrm{p}$.

SORESSI M. 2005 - Late Mousterian lithic technology: its implications for the place of the emergence of behavioural modernity and the relationship between behavioural modernity and biological modernity. In: D'Errico F., Backwell L. (Eds.), From tools to symbols: from early hominids to modern humans. Johannesburg: Witwatersrand University Press, p. 389-417.

SORESSI M. et PELEGRIN J. 2007 - Le Châtelperronien et ses rapports avec le Moustérien. In : Vandermeersch B. et Maureille B. (Eds), Les Néandertaliens, biologie et cultures, Paris : CTHS, Documents préhistoriques, 23, p. 283-296.

TAVOSO A. 1978 - Le Paléolithique inférieur et moyen du Haut-Languedoc. Gisements des terrasses alluviales $d u$ Tarn, du Dadou, de l'Agout, du Sor et du Fresquel. Université de Provence : éditions du Laboratoires de Paléontologie Humaine et de Préhistoire, Études Quaternaires, mémoire 5, 404 p.

TEXIER J.-P. 2009 - Histoire géologique de sites préhistoriques du Périgord: une vision actualisée. La Micoque, la grotte Vaufrey, le Pech de l'Azé I et II, La Ferrassie, L'abri Castanet, Le Flageolet, Laugerie-Haute. Paris, éd. du CTHS 194 p. (Documents préhistoriques, 25).

THIÉBAUT C. 2005 - Le Moustérien à denticulés : variabilité ou diversité techno-économique ? Thèse de doctorat, Aix-Marseille 1, Université de Provence, 231p. $+635 p$.

THIÉBAUT C. 2007 - Le Moustérien à denticulés des années cinquante à nos jours : définition et caractérisation. Bull. de la Soc. Préhist. française, T. 104, n 3, p. 461-482.
THIÉBAUT C., MEIGNEN L. et LEVÊQUE F. 2009 - Les dernières occupations moustériennes de Saint-Césaire (Charente-Maritime, France) : diversité des techniques utilisées et comportements économiques pratiques. Bulletin de la Société Préhistorique Française, 106, 4, p. 691-714.

TURQ A. 1992 - Le Paléolithique inférieur et moyen entre les vallées de la Dordogne et du Lot, Thèse ND, Université de Bordeaux I, 2 vol., 780 p., ill., $n^{\circ} 778$.

TURQ A. 2000 - Le Paléolithique inférieur et moyen entre Dordogne et Lot. Paleo, Supplément $n^{\circ} 2,456 \mathrm{p}$.

TURQ A. et JAUBERT J. 2008 - Le contexte culturel moustérien. In : B. Vandermeersch, J.-J. Cleyet-Merle, J. Jaubert, B. Maureille et A. Turq (dir.) 2008. Première humanité : gestes funéraires des Néandertaliens. Réunion des musées nationaux, Paris, p. 25-37.

TURQ A. et MORALA A. 2013 (Séronie-Vivien M. et M.-R., coll.) 2013 - Inventaire des silicifications du Quercy et de ses marges et des marqueurs lithologiques du nord-est aquitain : synthèse des données. In : Jarry M., J.-Ph. Brugal et C. Ferrier (Dir.), Modalités d'occupation et exploitation des milieux au Paléolithique dans le Sud-Ouest de la France : l'exemple du Quercy, colloque 67, XVème congrès de l'UISPP, Lisbonne, 2006, suppl. n4, Paleo. p. 159-180.

TURQ A., ANTIGNAC G. et ROUSSEL P. 1999 - Les silicifications coniaciennes du Sarladais et $\mathrm{du}$ Gourdonnais : inventaire et implications archéologiques. Paleo, 11, p. 145-160.

TURQ A., DIBBLE H., FAIVRE J.-Ph., GOLDBERG P., McPHERRON Sh. J. P. et SANDGATHE D. 2008 - Le Moustérien du Périgord : quoi de neuf ? In : J. Jaubert, J.G. Bordes et I. Ortega (Eds.), Les sociétés du Paléolithique dans un Grand Sud-Ouest de la France : nouveaux gisements, nouveaux résultats, nouvelles méthodes. Actes des journées de la SPF à Bordeaux (24-25 novembre 2006), mémoire XLVII de la SPF, p. 83-93. 